Supporting Information

\title{
Programmable Aggregation of Artificial Cells with DNA Signals
}

Hengming Qiu ${ }^{1+}$, Feiran $\mathrm{Li}^{1+}$, Yancheng $\mathrm{Du}^{1+}$, Ruixin $\mathrm{Li}^{1}$, Ji Yeon Hyun², Sei Young Lee ${ }^{2 *}$, Jong Hyun Choi ${ }^{1 *}$

${ }^{1}$ School of Mechanical Engineering. Purdue University, West Lafayette, Indiana 47907, USA

${ }^{2}$ Department of Biomedical Engineering, Yonsei University, Wonju, Gangwon 26427, Republic of Korea

+These authors contributed equally to this work

*Corresponding author: jchoi@purdue.edu, syl235@yonsei.ac.kr

\section{Table of content}

1. Materials

- Origami design

2. Characterization

- AFM images of tubular and rectangular DNA origami

- FRET measurement of cap-pore binding

- Additional kinetic measurement

- Exo III activity inside a giant vesicle

- Immoblized giant vesicle shape change over time

- Reversible aggregation behavior using DNA signals

- FRET measurement of vesicle aggregation

3. References 


\section{Materials}

\section{Origami design}

(a)

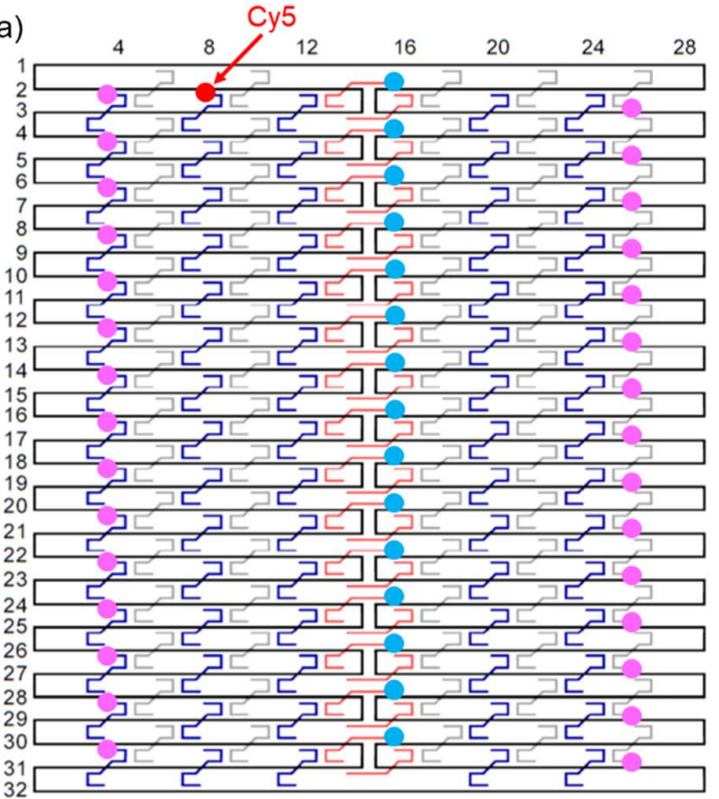

(d)

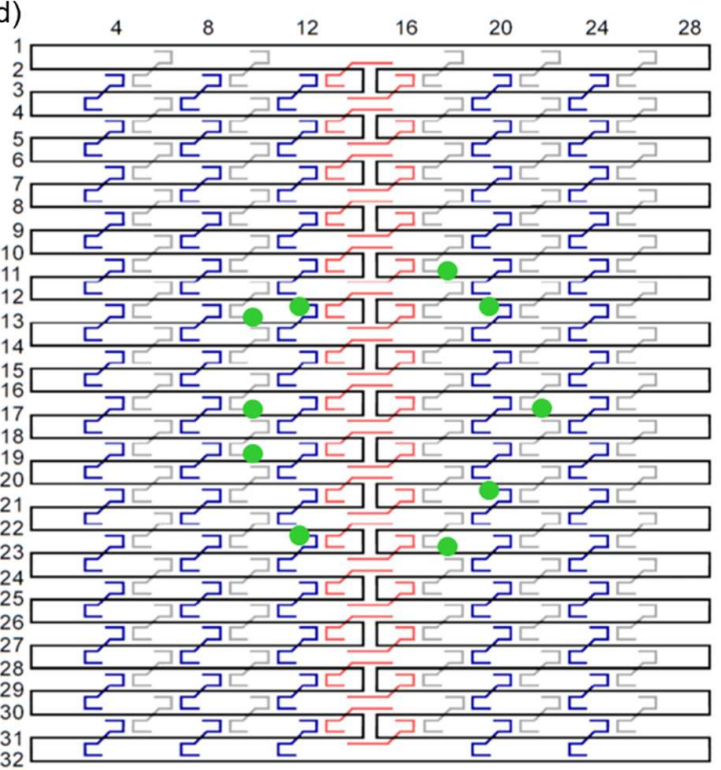

(g)

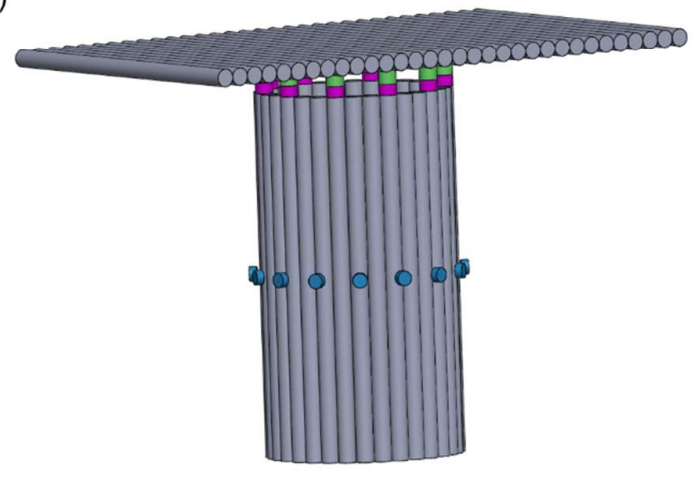

(b)

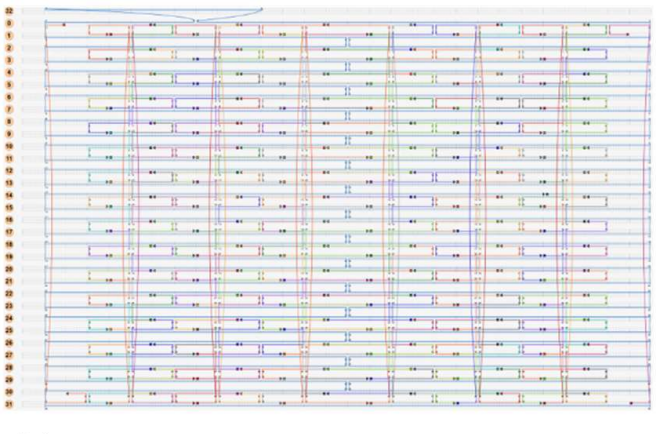

(c)

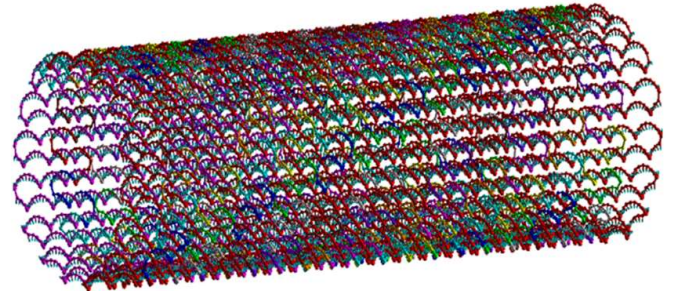

(e)

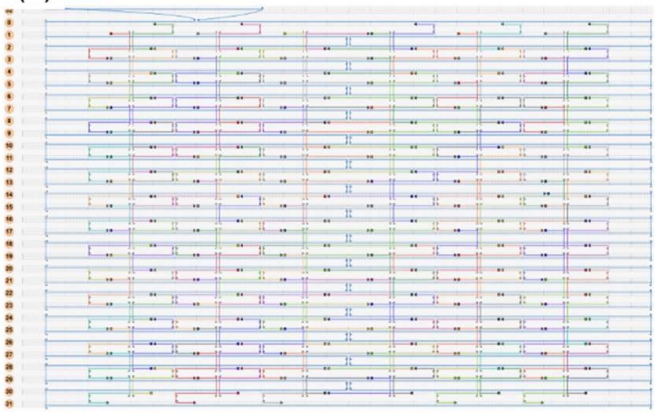

(f)

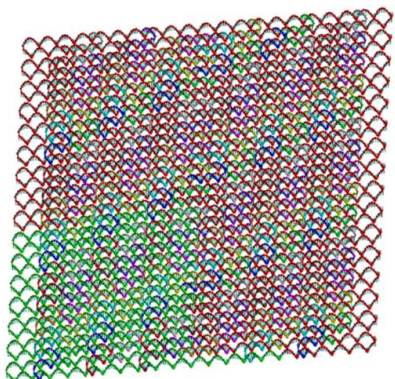


Figure S1. The origami designs and folding path diagrams for the tubular pore (a)-(c) and rectangular cap (d)-(f). The numbers on origami diagrams in (a) and (d) are used as marks for rows and columns in the origami designs. (a) Schematic and (b) caDNAno design of the 32helix origami tile which cyclizes to form a tubular pore, as shown in the schematic in (c). The staple strands are presented in Table S1. A set of staples (termed tubular staples, listed in the bottom of Table S1) are used to connect the top and bottom of the tile, leading to a cyclization and forming a tubular pore. These tubular staples are not shown in the schematic. A Cy5-DNA strand, used for fluorescence imaging in Figure 2a-b, is marked as red dot (shown in Figure S1a). A total of 30 staple strands (15 on each side), marked as pink dots, have a 16-nt singlestranded extension (on $5^{\prime}$ end) to connect the tubular pore with the rectangular cap. The extensions are marked with pink color in Table S1. The 15 staples marked in sky-blue are designed to link with cholesterol-DNA with a 27-nt extension (listed in Table S1). These staples help to embed the pores on the surfaces of GUVs. The sequences are marked with sky-blue color in Table S1. (d) Schematic and (e) caDNAno design of the DNA origami cap. The staple strands are presented in Table S2. Ten of the staples (indicated by green dots) have a 24-nt extension on 5' end. The 24-nt extension consists of two domains: 16-nt (TTCGGACAGAGTGACA) for binding the pore and 8-nt (AGTGCTGA) used as a toehold. (f) Schematic of a rectangular cap DNA origami. The extensions are presented in green in Table S2. Actual shape of the origami caps may experience minor curvature as noted by previous research. ${ }^{1-2}$ (g) Schematic of the tubular pore closed with the rectangular cap. The sky-blue dots represent the staples connected to cholesterol moieties. The pink and green dots denote the staple extensions for capping.

Table S1. Collection of staples for the tubular DNA origami pore. Blue, gray and red staples for the DNA origami, illustrated in Figure S1a. The numbers are used to show the positions of the staples. For example, [02,08] represents the staple inserted at row number 2 and column number 8 in the M13mp18 origami scaffold. Colored parts in the strands in the table correspond to the colored dots in Figure S1. For example, staple $[02,08]$ has a red '/5Cy5/' at the 5' end of the staple's sequence matching with the red dot in Figure S1a. Similar notations apply to pink extensions in staples. These extensions match with the pink dots in Figure S1a and are used as binding points on the pores. A total number of 15 sky-blue extensions correspond to the sky-blue dots in Figure S1a. These extensions are designed to bind with a cholesterol-DNA (TGGACGGCCGTCAACTGCGGCGTGTAA/3CholTEG/) for embedding the pores in the surfaces of GUVs. Tubular staples listed at the bottom of the table are designed to bind the upper and lower boundaries of the origami tile together, thus forming a tubular pore as shown in Figure S1C.

\begin{tabular}{|c|l|l|l|}
\hline \multicolumn{2}{|c|}{ Blue staples } & \multicolumn{2}{c|}{ Gray staples } \\
\hline Name & \multicolumn{1}{|c|}{ Sequence } & Name & \multicolumn{1}{c|}{ Sequence } \\
\hline \multirow{2}{*}[02,04]{} & $\begin{array}{l}\text { TGTCACTCTGTCCGAAGAACGGTACAGAA } \\
\text { CAATATTACCGAATACCTA }\end{array}$ & {$[03,05]$} & $\begin{array}{l}\text { GTAATATCCGCCAGAATCCTGAGAGTATAA } \\
\text { CG }\end{array}$ \\
\hline$[02,08]$ & $\begin{array}{l}\text { I5Cy5/TATAATCAGAACTCAAACTATCGGAT } \\
\text { GGATTA }\end{array}$ & {$[03,09]$} & $\begin{array}{l}\text { GAGTAGAAGTGAGGCCACCGAGTAGAGCG } \\
\text { GGC }\end{array}$ \\
\hline [02, 12] & $\begin{array}{l}\text { TGTCCATCGATTAGTAATAACATCACACGA } \\
\text { CC }\end{array}$ & {$[03,17]$} & $\begin{array}{l}\text { AAAATCCCTGAGTGTTGTTCCAGTCGATTT } \\
\text { AG }\end{array}$ \\
\hline
\end{tabular}




\begin{tabular}{|c|c|c|c|}
\hline$[02,20]$ & $\begin{array}{l}\text { AGAGTCCATTTGATGGTGGTTCCGAGAGG } \\
\text { CGG }\end{array}$ & {$[03,21]$} & $\begin{array}{l}\text { AAATCCTGCTATTAAAGAACGTGGAAGCAC } \\
\text { TA }\end{array}$ \\
\hline$[02,24]$ & $\begin{array}{l}\text { GTCAAAGGACGCTGGTTTGCCCCATTTTTC } \\
\text { TT }\end{array}$ & {$[03,25]$} & $\begin{array}{l}\text { TGTCACTCTGTCCGAAAGCGGTCCGCGAA } \\
\text { AAACCGTCTATCAAATCAA }\end{array}$ \\
\hline$[04,04]$ & $\begin{array}{l}\text { TGTCACTCTGTCCGAACATTTTGAATGCGC } \\
\text { GAACTGATAGAACCACCA }\end{array}$ & {$[05,05]$} & $\begin{array}{l}\text { AGTCTTTACGCTCAATCGTCTGAACCTTGC } \\
\text { TG }\end{array}$ \\
\hline$[04,08]$ & $\begin{array}{l}\text { TTTACATTAGACAATATTTTTGAACGGTCAG } \\
\mathrm{T}\end{array}$ & {$[05,09]$} & $\begin{array}{l}\text { CGTGGCACGGCAGATTCACCAGTCACTTG } \\
\text { CCT }\end{array}$ \\
\hline$[04,12]$ & $\begin{array}{l}\text { AGTAATAATTCTGACCTGAAAGCGACGCTG } \\
\text { AG }\end{array}$ & {$[05,17]$} & $\begin{array}{l}\text { TCCAGTCGCGGCCAACGCGCGGGGAAAT } \\
\text { CGGC }\end{array}$ \\
\hline$[04,20]$ & $\begin{array}{l}\text { TTTGCGTATTGCGTTGCGCTCACTGGGTAC } \\
\text { CG }\end{array}$ & {$[05,21]$} & $\begin{array}{l}\text { CACATTAATTGGGCGCCAGGGTGGGCAGG } \\
\text { CGA }\end{array}$ \\
\hline$[04,24]$ & $\begin{array}{l}\text { TTCACCAGGGGTGCCTAATGAGTGTAGCT } \\
\text { GTT }\end{array}$ & {$[05,25]$} & $\begin{array}{l}\text { TGTCACTCTGTCCGAAAAAGCCTGTGAGA } \\
\text { CGGGCAACAGCTTGCAGCA }\end{array}$ \\
\hline$[06,04]$ & $\begin{array}{l}\text { TGTCACTCTGTCCGAAGCAGAAGATGAGG } \\
\text { AAGGTTATCTATTAGAGCC }\end{array}$ & {$[07,05]$} & $\begin{array}{l}\text { AAAGGAATTAAAACAGAGGTGAGGTGGCT } \\
\text { ATT }\end{array}$ \\
\hline$[06,08]$ & $\begin{array}{l}\text { ATTAACACGGTCAGTTGGCAAATCTTTAGA } \\
\text { AG }\end{array}$ & {$[07,09]$} & $\begin{array}{l}\text { CAATATCTCGCCTGCAACAGTGCCTAAGAA } \\
\text { TA }\end{array}$ \\
\hline$[06,12]$ & $\begin{array}{l}\text { AGCCAGCAAACCTCAAATATCAAACAACTC } \\
\text { GT }\end{array}$ & {$[07,17]$} & $\begin{array}{l}\text { AAAACGACACTCTAGAGGATCCCCGCCCG } \\
\text { СTT }\end{array}$ \\
\hline$[06,20]$ & $\begin{array}{l}\text { AGCTCGAAGGGTTTTCCCAGTCACTCTGG } \\
\text { TGC }\end{array}$ & {$[07,21]$} & $\begin{array}{l}\text { TAACGCCATTCGTAATCATGGTCAAGCTAA } \\
\text { CT }\end{array}$ \\
\hline$[06,24]$ & $\begin{array}{l}\text { TCCTGTGTGTGCTGCAAGGCGATTGCCAT } \\
\text { TCA }\end{array}$ & {$[07,25]$} & $\begin{array}{l}\text { TGTCACTCTGTCCGAAAGGGGGATGAAAT } \\
\text { TGTTATCCGCTTAAAGTGT }\end{array}$ \\
\hline$[08,04]$ & $\begin{array}{l}\text { TGTCACTCTGTCCGAAGTCAATAGCTGATT } \\
\text { ATCAGATGATATTATACT }\end{array}$ & {$[09,05]$} & $\begin{array}{l}\text { TCATATTCATAATACATTTGAGGAAACAGTT } \\
\text { G }\end{array}$ \\
\hline$[08,08]$ & $\begin{array}{l}\text { TATTAGACCCACCAGAAGGAGCGGCTACC } \\
\text { ATA }\end{array}$ & {$[09,09]$} & $\begin{array}{l}\text { CAAAGAAATTTACAAACAATTCGACCCTCA } \\
\text { AT }\end{array}$ \\
\hline$[08,12]$ & $\begin{array}{l}\text { ATTAAATCGAGTAACATTATCATTGAAATAA } \\
\text { A }\end{array}$ & {$[09,17]$} & $\begin{array}{l}\text { CGACGACAGCTTTCCGGCACCGCTGACGT } \\
\text { TGT }\end{array}$ \\
\hline$[08,20]$ & $\begin{array}{l}\text { CGGAAACCCGTGCATCTGCCAGTTTAATTC } \\
\text { GC }\end{array}$ & {$[09,21]$} & $\begin{array}{l}\text { ATCGTAACAGGCAAAGCGCCATTCAAGTT } \\
\text { GGG }\end{array}$ \\
\hline$[08,24]$ & $\begin{array}{l}\text { GGCTGCGCGGTCACGTTGGTGTAGTCATC } \\
\text { AAC }\end{array}$ & {$[09,25]$} & $\begin{array}{l}\text { TGTCACTCTGTCCGAAATGGGATAAACTGT } \\
\text { TGGGAAGGGCCTGGCGAA }\end{array}$ \\
\hline$[10,04]$ & $\begin{array}{l}\text { TGTCACTCTGTCCGAATCTGAATAAAGTTA } \\
\text { CAAAATCGCGCAAAAGAA }\end{array}$ & {$[11,05]$} & $\begin{array}{l}\text { TGAATACCATGGAAGGGTTAGAACAATTAT } \\
\text { CA }\end{array}$ \\
\hline$[10,08]$ & $\begin{array}{l}\text { TCAAAATTAATAACGGATTCGCCTACAAAA } \\
\text { TT }\end{array}$ & {$[11,09]$} & $\begin{array}{l}\text { GGAGAAACATTTGCACGTAAAACATTGCG } \\
\text { GAA }\end{array}$ \\
\hline$[10,12]$ & $\begin{array}{l}\text { GAAATTGCACAGTAACAGTACCTTAATTAC } \\
\text { CT }\end{array}$ & {$[11,17]$} & $\begin{array}{l}\text { CATTAAATGGAACGCCATCAAAAATGAGGG } \\
\text { GA }\end{array}$ \\
\hline$[10,20]$ & $\begin{array}{l}\text { GTCTGGCCACGTTAATATTTTGTTGGTCAT } \\
\text { TG }\end{array}$ & {$[11,21]$} & $\begin{array}{l}\text { AATTGTAATTCCTGTAGCCAGCTTATGGGC } \\
\text { GC }\end{array}$ \\
\hline
\end{tabular}




\begin{tabular}{|c|c|c|c|}
\hline$[10,24]$ & $\begin{array}{l}\text { ATTAAATGGAAGATTGTATAAGCAGAATCG } \\
\text { AT }\end{array}$ & {$[11,25]$} & $\begin{array}{l}\text { TGTCACTCTGTCCGAAAAAAACAGTGAGC } \\
\text { GAGTAACAACCTGACCGTA }\end{array}$ \\
\hline$[12,04]$ & $\begin{array}{l}\text { TGTCACTCTGTCCGAAGATGATGAGCGATA } \\
\text { GCTTAGATTAAAAATCAT }\end{array}$ & {$[13,05]$} & $\begin{array}{l}\text { AAAACATAAACAAACATCAAGAAAGATTGC } \\
\text { TT }\end{array}$ \\
\hline$[12,08]$ & $\begin{array}{l}\text { AATTACATATTAATTTTCCCTTAGCCTCCGG } \\
\text { C }\end{array}$ & {$[13,09]$} & $\begin{array}{l}\text { CGCTATTATTAACAATTTCATTTGTTACATC } \\
\text { G }\end{array}$ \\
\hline$[12,12]$ & $\begin{array}{l}\text { TTTTTAATAATAACCTTGCTTCTGGTAAATG } \\
\text { C }\end{array}$ & {$[13,17]$} & $\begin{array}{l}\text { TGATAAATTCTACAAAGGCTATCAAAAATTC } \\
\text { G }\end{array}$ \\
\hline$[12,20]$ & $\begin{array}{l}\text { CCTGAGAGAATATGATATTCAACCTAATAC } \\
\text { TT }\end{array}$ & {$[13,21]$} & $\begin{array}{l}\text { TCACCATCTCTGGAGCAAACAAGAAATATT } \\
\text { TA }\end{array}$ \\
\hline$[12,24]$ & $\begin{array}{l}\text { GAACGGTATGAGAAAGGCCGGAGACGCAA } \\
\text { GGA }\end{array}$ & {$[13,25]$} & $\begin{array}{l}\text { TGTCACTCTGTCCGAACAAAAGGGATCGTA } \\
\text { AAACTAGCATAAAGCCCC }\end{array}$ \\
\hline$[14,04]$ & $\begin{array}{l}\text { TGTCACTCTGTCCGAAAGGTCTGACGTGT } \\
\text { GATAAATAAGGTACTAGAA }\end{array}$ & {$[15,05]$} & $\begin{array}{l}\text { ATACCGACGAGACTACCTTTTTAAAATCCT } \\
\text { TG }\end{array}$ \\
\hline$[14,08]$ & $\begin{array}{l}\text { TTAGGTTGCTGACCTAAATTTAATTTATACA } \\
\text { A }\end{array}$ & {$[15,09]$} & $\begin{array}{l}\text { TTCATCTTGGTTATATAACTATATTAAATCG } \\
\mathrm{T}\end{array}$ \\
\hline$[14,12]$ & $\begin{array}{l}\text { TGATGCAATTTTTCAAATATATTTCTCAACA } \\
\text { G }\end{array}$ & {$[15,17]$} & $\begin{array}{l}\text { AATAAAGCAAACATTATGACCCTGGTTCTA } \\
\text { GC }\end{array}$ \\
\hline$[14,20]$ & $\begin{array}{l}\text { TTGCGGGAGGCAAAGAATTAGCAAGTAGA } \\
\text { TTT }\end{array}$ & {$[15,21]$} & $\begin{array}{l}\text { ACAGGCAAGAAGCCTTTATTTCAACAGTCA } \\
\text { AA }\end{array}$ \\
\hline$[14,24]$ & $\begin{array}{l}\text { TAAAAATTTAGCATTAACATCCAACAAATG } \\
\text { GT }\end{array}$ & {$[15,25]$} & $\begin{array}{l}\text { TGTCACTCTGTCCGAAAATAGTAGTTTAGA } \\
\text { ACCCTCATATTAAAGATT }\end{array}$ \\
\hline$[16,04]$ & $\begin{array}{l}\text { TGTCACTCTGTCCGAAAAAGCCTGAAAGTA } \\
\text { ATTCTGTCCAGCAGAACG }\end{array}$ & {$[17,05]$} & $\begin{array}{l}\text { CAAAAGGTTTTAGTATCATATGCGGGTTTG } \\
\text { AA }\end{array}$ \\
\hline$[16,08]$ & $\begin{array}{l}\text { ATTCTTACTAATAAGAGAATATAAGTCCTGA } \\
\text { A }\end{array}$ & {$[17,09]$} & $\begin{array}{l}\text { CGAGCCAGCAGTATAAAGCCAACGTAGTT } \\
\text { AAT }\end{array}$ \\
\hline$[16,12]$ & $\begin{array}{l}\text { TAGGGCTTATGTAATTTAGGCAGAATTTAC } \\
\text { GA }\end{array}$ & {$[17,17]$} & $\begin{array}{l}\text { TACGGTGTCCAATTCTGCGAACGAAATTAA } \\
\text { GC }\end{array}$ \\
\hline$[16,20]$ & $\begin{array}{l}\text { AGTTTGACCATGTTTTAAATATGCTAATTCG } \\
\text { A }\end{array}$ & {$[17,21]$} & $\begin{array}{l}\text { TAGCTCAACATTAGATACATTTCGTAAATCA } \\
\text { T }\end{array}$ \\
\hline$[16,24]$ & $\begin{array}{l}\text { CAATAACCGCTTAATTGCTGAATAGCAAAC } \\
\text { TC }\end{array}$ & {$[17,25]$} & $\begin{array}{l}\text { TGTCACTCTGTCCGAAGGCTTAGATGTTTA } \\
\text { GCTATATTTTATTCTACT }\end{array}$ \\
\hline$[18,04]$ & $\begin{array}{l}\text { TGTCACTCTGTCCGAACGCCTGTTTTCATC } \\
\text { GTAGGAATCAAGAAGGCT }\end{array}$ & {$[19,05]$} & $\begin{array}{l}\text { TTTTTATTTATCAACAATAGATAAAGTACCG } \\
\text { A }\end{array}$ \\
\hline$[18,08]$ & $\begin{array}{l}\text { CAAGAAAACACTCATCGAGAACAAGCGTTT } \\
\text { TA }\end{array}$ & {$[19,09]$} & $\begin{array}{l}\text { AAGTACCGATAATATCCCATCCTAGGCATT } \\
\text { TT }\end{array}$ \\
\hline$[18,12]$ & $\begin{array}{l}\text { GCATGTAGCATTCCAAGAACGGGTTTTTGA } \\
\text { AG }\end{array}$ & {$[19,17]$} & $\begin{array}{l}\text { GCGGATTGTTCAAATATCGCGTTTAACTAA } \\
\text { AG }\end{array}$ \\
\hline$[18,20]$ & $\begin{array}{l}\text { GCTTCAAACTGACTATTATAGTCAGCCAGA } \\
\text { GG }\end{array}$ & {$[19,21]$} & $\begin{array}{l}\text { TCTTTACCGCGAACCAGACCGGAATAATG } \\
\text { CTG }\end{array}$ \\
\hline$[18,24]$ & $\begin{array}{l}\text { CAACAGGTAATGACCATAAATCAAGGATAG } \\
\text { CG }\end{array}$ & {$[19,25]$} & $\begin{array}{l}\text { TGTCACTCTGTCCGAAAAAACGAGCAGGA } \\
\text { TTAGAGAGTACTTGCGGAT }\end{array}$ \\
\hline
\end{tabular}




\begin{tabular}{|c|c|c|c|}
\hline$[20,04]$ & $\begin{array}{l}\text { TGTCACTCTGTCCGAATATCCGGTAATAAA } \\
\text { CAGCCATATTTGTTTAAC }\end{array}$ & {$[21,05]$} & $\begin{array}{l}\text { AGTTACAAATTCTAAGAACGCGAGGCAAG } \\
\text { CCG }\end{array}$ \\
\hline$[20,08]$ & $\begin{array}{l}\text { GCGAACCTCGTCTTTCCAGAGCCTTACAG } \\
\text { AGA }\end{array}$ & {$[21,09]$} & $\begin{array}{l}\text { CTAACGAGCCCGACTTGCGGGAGGATTAA } \\
\text { ACC }\end{array}$ \\
\hline$[20,12]$ & $\begin{array}{l}\text { CCTTAAATATTTTATCCTGAATCTCATTAGA } \\
\text { C }\end{array}$ & {$[21,17]$} & $\begin{array}{l}\text { TTTACCAGTTGCAAAAGAAGTTTTGAAGCA } \\
\text { AA }\end{array}$ \\
\hline$[20,20]$ & $\begin{array}{l}\text { GGGTAATAAGAGCAACACTATCATCGTTGG } \\
\text { GA }\end{array}$ & {$[21,21]$} & $\begin{array}{l}\text { GCATAGTAGTAAAATGTTTAGACTAAATCA } \\
\text { GG }\end{array}$ \\
\hline$[20,24]$ & $\begin{array}{l}\text { TCCAATACCATAACGCCAAAAGGAAACTAA } \\
\text { CG }\end{array}$ & {$[21,25]$} & $\begin{array}{l}\text { TGTCACTCTGTCCGAATGCAGATATGCGG } \\
\text { AATCGTCATAACAGTTCAG }\end{array}$ \\
\hline$[22,04]$ & $\begin{array}{l}\text { TGTCACTCTGTCCGAAGTCAAAAAAAACAA } \\
\text { TGAAATAGCAAGTAAGCA }\end{array}$ & {$[23,05]$} & $\begin{array}{l}\text { AGAGCAAGTGAAAATAGCAGCCTTAATTTG } \\
\text { CC }\end{array}$ \\
\hline$[22,08]$ & $\begin{array}{l}\text { GAATAACAAGAATTGAGTTAAGCCGGAAAC } \\
\text { CG }\end{array}$ & {$[23,09]$} & $\begin{array}{l}\text { AACCCACATAAAAACAGGGAAGCGTACCA } \\
\text { ACG }\end{array}$ \\
\hline$[22,12]$ & $\begin{array}{l}\text { GGGAGAATATTGAGCGCTAATATCCCCAAA } \\
\text { AG }\end{array}$ & {$[23,17]$} & $\begin{array}{l}\text { ATCATTGTATTATACCAGTCAGGAAACCCT } \\
\text { CG }\end{array}$ \\
\hline$[22,20]$ & $\begin{array}{l}\text { AGAAAAATTGAGATGGTTTAATTTGGCGCA } \\
\text { TA }\end{array}$ & {$[23,21]$} & $\begin{array}{l}\text { ATTGGGCTCTACGTTAATAAAACGATTACG } \\
\text { AG }\end{array}$ \\
\hline$[22,24]$ & $\begin{array}{l}\text { GAACAACAGAGAAACACCAGAACGAATCTT } \\
\text { GA }\end{array}$ & {$[23,25]$} & $\begin{array}{l}\text { TGTCACTCTGTCCGAAGCCCTGACTTATTA } \\
\text { CAGGTAGAAATCAACTAA }\end{array}$ \\
\hline$[24,04]$ & $\begin{array}{l}\text { TGTCACTCTGTCCGAAGATAGCCGTAAGTT } \\
\text { TATTTTGTCAGCCAAAGA }\end{array}$ & {$[25,05]$} & $\begin{array}{l}\text { CCACGGAAAACAAAGTTACCAGAACAATAA } \\
\text { TA }\end{array}$ \\
\hline$[24,08]$ & $\begin{array}{l}\text { AGGAAACGACATATAAAAGAAACGGAGGG } \\
\text { AGG }\end{array}$ & {$[25,09]$} & $\begin{array}{l}\text { AGGTGGCACAATAATAACGGAATAAGAGA } \\
\text { GAT }\end{array}$ \\
\hline$[24,12]$ & $\begin{array}{l}\text { AACTGGCACAAACGTAGAAAATACTTCATT } \\
\text { AA }\end{array}$ & {$[25,17]$} & $\begin{array}{l}\text { TAAGGGAAAACGGTGTACAGACCACAACT } \\
\text { TTA }\end{array}$ \\
\hline$[24,20]$ & $\begin{array}{l}\text { GGCTGGCTGAACGAGGCGCAGACGCGAA } \\
\text { AGAG }\end{array}$ & {$[25,21]$} & $\begin{array}{l}\text { CTTAGCCGGACCTTCATCAAGAGTAGTAGT } \\
\text { AA }\end{array}$ \\
\hline$[24,24]$ & $\begin{array}{l}\text { CAAGAACCAAATCCGCGACCTGCTATCTTT } \\
\text { GA }\end{array}$ & {$[25,25]$} & $\begin{array}{l}\text { TGTCACTCTGTCCGAATTGTGTCGGGATAT } \\
\text { TCATTACCCATAAGGCTT }\end{array}$ \\
\hline$[26,04]$ & $\begin{array}{l}\text { TGTCACTCTGTCCGAACAAAAGGGAACCAT } \\
\text { CGATAGCAGCCTTTAGCG }\end{array}$ & {$[27,05]$} & $\begin{array}{l}\text { ACCAATGACGACATTCAACCGATTCAAAGA } \\
\text { CA }\end{array}$ \\
\hline$[26,08]$ & $\begin{array}{l}\text { GAAGGTAAACCATTAGCAAGGCCGGCATT } \\
\text { TTC }\end{array}$ & {$[27,09]$} & $\begin{array}{l}\text { GCACCATTATATTGACGGAAATTAATACAT } \\
\text { AA }\end{array}$ \\
\hline$[26,12]$ & $\begin{array}{l}\text { AGGTGAATTTAGAGCCAGCAAAATTGCCAT } \\
\text { СT }\end{array}$ & {$[27,17]$} & $\begin{array}{l}\text { AGTTTCCAAGGCACCAACCTAAAAGTCAAT } \\
\text { CA }\end{array}$ \\
\hline$[26,20]$ & $\begin{array}{l}\text { GCAAAAGAGGACTAAAGACTTTTTTTGACAA } \\
\text { CA }\end{array}$ & {$[27,21]$} & $\begin{array}{l}\text { GGCTTTGAATACACTAAAACACTCCCATGT } \\
\text { TA }\end{array}$ \\
\hline$[26,24]$ & $\begin{array}{l}\text { CCCCCAGCAACGAGGGTAGCAACGTATTC } \\
\text { GGT }\end{array}$ & {$[27,25]$} & $\begin{array}{l}\text { TGTCACTCTGTCCGAAAGCATCGGGATTAT } \\
\text { ACCAAGCGCGCTGATAAA }\end{array}$ \\
\hline$[28,04]$ & $\begin{array}{l}\text { TGTCACTCTGTCCGAATCAGACTGCCACCA } \\
\text { GAACCACCACGGCAGGTC }\end{array}$ & {$[29,05]$} & $\begin{array}{l}\text { CAGAGCCGTAGCGCGTTTTCATCGGAAAC } \\
\text { GTC }\end{array}$ \\
\hline
\end{tabular}




\begin{tabular}{|c|c|c|c|}
\hline$[28,08]$ & $\begin{array}{l}\text { GGTCATAGCGCCACCCTCAGAGCCAACAA } \\
\text { ATA }\end{array}$ & {$[29,09]$} & $\begin{array}{l}\text { CTCAGAACCCCCCTTATTAGCGTTCACCAG } \\
\text { TA }\end{array}$ \\
\hline$[28,12]$ & $\begin{array}{l}\text { TTTCATAAACCGCCTCCCTCAGAGAAGCG } \\
\text { CAG }\end{array}$ & {$[29,17]$} & $\begin{array}{l}\text { GCTTGCTTATAGTTGCGCCGACAACATGA } \\
\text { GGA }\end{array}$ \\
\hline$[28,20]$ & $\begin{array}{l}\text { ACCATCGCGCCTTTAATTGTATCGTTAGTA } \\
\text { AA }\end{array}$ & {$[29,21]$} & $\begin{array}{l}\text { CAAAAGGACCACGCATAACCGATAGCTAC } \\
\text { AGA }\end{array}$ \\
\hline$[28,24]$ & $\begin{array}{l}\text { CGCTGAGGTGAAAATCTCCAAAAATAAACA } \\
\text { AC }\end{array}$ & {$[29,25]$} & $\begin{array}{l}\text { TGTCACTCTGTCCGAATTTCACGTCTTGCA } \\
\text { GGGAGTTAAACGAAAGAC }\end{array}$ \\
\hline$[30,04]$ & $\begin{array}{l}\text { TGTCACTCTGTCCGAAAGACGATTCGTATA } \\
\text { AACAGTTAATAAACATGA }\end{array}$ & {$[31,05]$} & $\begin{array}{l}\text { ACAGTGCCGGCCTTGATATTCACAACCAC } \\
\text { СCT }\end{array}$ \\
\hline$[30,08]$ & $\begin{array}{l}\text { AATCCTCATTAACGGGGTCAGTGCCAAGA } \\
\text { GAA }\end{array}$ & {$[31,09]$} & $\begin{array}{l}\text { AATAAGTTTTAAAGCCAGAATGGACCGCCA } \\
\text { CC }\end{array}$ \\
\hline$[30,12]$ & $\begin{array}{l}\text { TCTCTGAATTGATGATACAGGAGTTCAGTA } \\
\text { CC }\end{array}$ & {$[31,17]$} & $\begin{array}{l}\text { ACAGACAGGTCGTCTTTCCAGACGGTTTAT } \\
\text { CA }\end{array}$ \\
\hline$[30,20]$ & $\begin{array}{l}\text { TGAATTTTAAACTACAACGCCTGTCACCGT } \\
\text { AC }\end{array}$ & {$[31,21]$} & $\begin{array}{l}\text { ACCAGTACCTGTATGGGATTTTGCAAAGGC } \\
\text { TC }\end{array}$ \\
\hline$[30,24]$ & $\begin{array}{l}\text { TTTCAACATGTACCGTAACACTGATCAGAA } \\
\text { CC }\end{array}$ & {$[31,25]$} & $\begin{array}{l}\text { TGTCACTCTGTCCGAAGGAACCCAGTTTCA } \\
\text { GCGGAGTGAGAATAATTT }\end{array}$ \\
\hline \multicolumn{4}{|c|}{ Red staples } \\
\hline Name & Sequence & Name & Sequence \\
\hline$[02,16]$ & $\begin{array}{l}\text { GATAGGGTTTATAAATCAAAAGAAGTAGCA } \\
\text { ATTTACACGCCGCAGTTGACGGCCGTCCA }\end{array}$ & {$[17,13]$} & $\begin{array}{l}\text { ACGCCAACAATTGAGAATCGCCATATATAA } \\
\text { CA }\end{array}$ \\
\hline$[03,13]$ & $\begin{array}{l}\text { ACTTCTTTACGCAAATTAACCGTTTAGCCC } \\
\text { GA }\end{array}$ & {$[18,16]$} & $\begin{array}{l}\text { CGAAAGACCATCAAAAAGATTAAGGGCTGT } \\
\text { CTTTACACGCCGCAGTTGACGGCCGTCCA }\end{array}$ \\
\hline$[04,16]$ & $\begin{array}{l}\text { TAATGAATGGAAACCTGTCGTGCCAACAGA } \\
\text { GATTACACGCCGCAGTTGACGGCCGTCCA }\end{array}$ & {$[19,13]$} & $\begin{array}{l}\text { TTCCTTATAAACCAATCAATAATCAGGAAG } \\
\text { CC }\end{array}$ \\
\hline$[05,13]$ & $\begin{array}{l}\text { TAGAACCCAAGGGACATTCTGGCCAGCTG } \\
\text { CAT }\end{array}$ & {$[20,16]$} & $\begin{array}{l}\text { AGAGGCTTACGACGATAAAAACCATTTGCA } \\
\text { CCTTACACGCCGCAGTTGACGGCCGTCCA }\end{array}$ \\
\hline$[06,16]$ & $\begin{array}{l}\text { GCAGGTCGGGCCAGTGCCAAGCTTAAGCA } \\
\text { TCATTACACGCCGCAGTTGACGGCCGTCC } \\
\text { A }\end{array}$ & {$[21,13]$} & $\begin{array}{l}\text { CAGCTACACAAGATTAGTTGCTATAAATAG } \\
\text { CG }\end{array}$ \\
\hline$[07,13]$ & $\begin{array}{l}\text { CCTTGCTGGCAAATGAAAAATCTAGCATGC } \\
\text { СT }\end{array}$ & {$[22,16]$} & $\begin{array}{l}\text { ACTGGCTCGAATTACCTTATGCGAACAAAG } \\
\text { TCTTACACGCCGCAGTTGACGGCCGTCCA }\end{array}$ \\
\hline$[08,16]$ & $\begin{array}{l}\text { TCCAGCCAGTATCGGCCTCAGGAATTAATT } \\
\text { TTTTACACGCCGCAGTTGACGGCCGTCCA }\end{array}$ & {$[23,13]$} & $\begin{array}{l}\text { AGAGGGTATAACTGAACACCCTGATTTTAAA } \\
\text { GA }\end{array}$ \\
\hline$[09,13]$ & $\begin{array}{l}\text { AAAAGTTTCTTTGCCCGAACGTTAGATCGC } \\
\text { AC }\end{array}$ & {$[24,16]$} & $\begin{array}{l}\text { GACAGATGCCGAACTGACCAACTTTTACG } \\
\text { CAGTTACACGCCGCAGTTGACGGCCGTCC } \\
\text { A }\end{array}$ \\
\hline$[10,16]$ & $\begin{array}{l}\text { AACCAATATTTTGTTAAATCAGCTAACGTCA } \\
\text { GTTACACGCCGCAGTTGACGGCCGTCCA }\end{array}$ & {$[25,13]$} & $\begin{array}{l}\text { TATGTTAGTGATTAAGACTCCTTATGAAAG } \\
\text { AG }\end{array}$ \\
\hline$[11,13]$ & $\begin{array}{l}\text { ATGAATATGTAGATTTTCAGGTTTCATTTTT } \\
\mathrm{T}\end{array}$ & {$[26,16]$} & $\begin{array}{l}\text { CACTACGATTAAACGGGTAAAATATTGAGC } \\
\text { CATTACACGCCGCAGTTGACGGCCGTCCA }\end{array}$ \\
\hline
\end{tabular}




\begin{tabular}{|c|c|c|c|}
\hline$[12,16]$ & $\begin{array}{l}\text { TTGAGAGATAATGCCGGAGAGGGTTCAAT } \\
\text { ATATTACACGCCGCAGTTGACGGCCGTCC } \\
\text { A }\end{array}$ & {$[27,13]$} & $\begin{array}{l}\text { TTTGGGAATATCACCGTCACCGACCGTAAT } \\
\text { GC }\end{array}$ \\
\hline$[13,13]$ & $\begin{array}{l}\text { TGTGAGTGGGAAACAGTACATAAAAGCTAT } \\
\text { TT }\end{array}$ & {$[28,16]$} & $\begin{array}{l}\text { TGATACCGTCGAGGTGAATTTCTTCAGAGC } \\
\text { CATTACACGCCGCAGTTGACGGCCGTCCA }\end{array}$ \\
\hline$[14,16]$ & $\begin{array}{l}\text { TGTACCAACTCAGAGCATAAAGCTAAGAAC } \\
\text { GCTTACACGCCGCAGTTGACGGCCGTCCA }\end{array}$ & {$[29,13]$} & $\begin{array}{l}\text { CCACCGGATCAAAATCACCGGAACAAACA } \\
\text { GCT }\end{array}$ \\
\hline$[15,13]$ & $\begin{array}{l}\text { GAGAAAACATCCAATCGCAAGACAAAATCG } \\
\text { GT }\end{array}$ & {$[30,16]$} & $\begin{array}{l}\text { AAAGTTTTCCCTCATAGTTAGCGTGCGTCA } \\
\text { TATTACACGCCGCAGTTGACGGCCGTCCA }\end{array}$ \\
\hline$[16,16]$ & $\begin{array}{l}\text { GTTGATTCCTGGAAGTTTCATTCCATTTAA } \\
\text { CATTACACGCCGCAGTTGACGGCCGTCCA }\end{array}$ & {$[31,13]$} & $\begin{array}{l}\text { CATGGCTTTTTACCGTTCCAGTAAAACGAT } \\
\text { CT }\end{array}$ \\
\hline \multicolumn{4}{|c|}{ Tubular staples } \\
\hline Name & Sequence & Name & Sequence \\
\hline$[1,2]$ & $\begin{array}{l}\text { AACAGGAGGGAACCTATTATTCTGGCCCC } \\
\text { CTG }\end{array}$ & {$[32,4]$} & $\begin{array}{l}\text { AAGTATTATCGTTAGAATCAGAGCTTAGAC } \\
\text { AG }\end{array}$ \\
\hline$[1,5]$ & $\begin{array}{l}\text { TGCTTTCCAGAGGCTGAGACTCCTCTTGA } \\
\text { GTA }\end{array}$ & {$[32,8]$} & $\begin{array}{l}\text { GGATTAGGGCTGGCAAACGAGCACAGTGT } \\
\text { TTT }\end{array}$ \\
\hline$[1,10]$ & $\begin{array}{l}\text { GCTAGGGCATTAGCGGGGTTTTGCGTACT } \\
\text { GGT }\end{array}$ & {$[32,12]$} & $\begin{array}{l}\text { AGGCGGATGGGAAGAAAGCGAAAGAAAGA } \\
\text { GTC }\end{array}$ \\
\hline$[1,14]$ & $\begin{array}{l}\text { GAAAGGAAAAGTGCCGTCGAGAGGGTTGA } \\
\text { TAT }\end{array}$ & {$[32,16]$} & $\begin{array}{l}\text { AAGTATAGGGGGAAAGCCGGCGAACGTG } \\
\text { GCGA }\end{array}$ \\
\hline$[1,18]$ & $\begin{array}{l}\text { AGCTTGACCCCGGAATAGGTGTATAGCATT } \\
\text { CC }\end{array}$ & {$[32,20]$} & $\begin{array}{l}\text { TCAGGAGGCCCTAAAGGGAGCCCCTTGGA } \\
\text { ACA }\end{array}$ \\
\hline$[1,22]$ & $\begin{array}{l}\text { AATCGGAATTTAGTACCGCCACCCGTTTCG } \\
\text { TC }\end{array}$ & {$[32,24]$} & $\begin{array}{l}\text { GCCACCCTGGGTCGAGGTGCCGTAACTCC } \\
\text { AAC }\end{array}$ \\
\hline$[1,26]$ & $\begin{array}{l}\text { GTTTTTTGCAGAACCGCCACCCTCGCCCA } \\
\text { ATA }\end{array}$ & {$[32,28]$} & $\begin{array}{l}\text { CACCCTCATACGTGAACCATCACCCAGGG } \\
\text { CGA }\end{array}$ \\
\hline
\end{tabular}

Table S2. Collection of staples for the rectangular origami cap. Blue, gray and red staples for the DNA origami, illustrated in Figure S1d. Green sequences in the strands in the table correspond to the green dots and are used as binding spots on the caps.

\begin{tabular}{|c|l|l|l|}
\hline \multicolumn{2}{|c|}{ Blue staples } & \multicolumn{2}{c|}{ Gray staples } \\
\hline Name & \multicolumn{1}{|c|}{ Sequence } & Name & \multicolumn{1}{c|}{ Sequence } \\
\hline$[02,04]$ & $\begin{array}{l}\text { GAACGGTACAGAACAATATTACCGAATACC } \\
\text { TA }\end{array}$ & {$[03,05]$} & $\begin{array}{l}\text { GTAATATCCGCCAGAATCCTGAGAGTATAA } \\
\text { CG }\end{array}$ \\
\hline$[02,08]$ & $\begin{array}{l}\text { TATAATCAGAACTCAAACTATCGGATGGAT } \\
\text { TA }\end{array}$ & {$[03,09]$} & $\begin{array}{l}\text { GAGTAGAAGTGAGGCCACCGAGTAGAGCG } \\
\text { GGC }\end{array}$ \\
\hline$[02,12]$ & $\begin{array}{l}\text { TGTCCATCGATTAGTAATAACATCACACGA } \\
\text { CC }\end{array}$ & {$[03,17]$} & $\begin{array}{l}\text { AAAATCCCTGAGTGTTGTTCCAGTCGATTT } \\
\text { AG }\end{array}$ \\
\hline$[02,20]$ & $\begin{array}{l}\text { AGAGTCCATTTGATGGTGGTTCCGAGAGG } \\
\text { CGG }\end{array}$ & $\begin{array}{l}\text { AAATCCTGCTATTAAAGAACGTGGAAGCAC } \\
\text { TA }\end{array}$ \\
\hline$[02,24]$ & $\begin{array}{l}\text { GTCAAAGGACGCTGGTTTGCCCCATTTTTC } \\
{[\text { TT }}\end{array}$ & $\begin{array}{l}\text { AGCGGTCCGCGAAAAACCGTCTATCAAAT } \\
\text { CAA }\end{array}$ \\
\hline
\end{tabular}




\begin{tabular}{|c|c|c|c|}
\hline$[04,04]$ & $\begin{array}{l}\text { CATTTTGAATGCGCGAACTGATAGAACCAC } \\
\text { CA }\end{array}$ & {$[05,05]$} & $\begin{array}{l}\text { AGTCTTTACGCTCAATCGTCTGAACCTTGC } \\
\text { TG }\end{array}$ \\
\hline$[04,08]$ & $\begin{array}{l}\text { TTTACATTAGACAATATTTTTGAACGGTCAG } \\
\text { T }\end{array}$ & {$[05,09]$} & $\begin{array}{l}\text { CGTGGCACGGCAGATTCACCAGTCACTTG } \\
\text { СCT }\end{array}$ \\
\hline$[04,12]$ & $\begin{array}{l}\text { AGTAATAATTCTGACCTGAAAGCGACGCTG } \\
\text { AG }\end{array}$ & {$[05,17]$} & $\begin{array}{l}\text { TCCAGTCGCGGCCAACGCGCGGGGAAAT } \\
\text { CGGC }\end{array}$ \\
\hline$[04,20]$ & $\begin{array}{l}\text { TTTGCGTATTGCGTTGCGCTCACTGGGTAC } \\
\text { CG }\end{array}$ & {$[05,21]$} & $\begin{array}{l}\text { CACATTAATTGGGCGCCAGGGTGGGCAGG } \\
\text { CGA }\end{array}$ \\
\hline$[04,24]$ & $\begin{array}{l}\text { TTCACCAGGGGTGCCTAATGAGTGTAGCT } \\
\text { GTT }\end{array}$ & {$[05,25]$} & $\begin{array}{l}\text { AAAGCCTGTGAGACGGGCAACAGCTTGCA } \\
\text { GCA }\end{array}$ \\
\hline$[06,04]$ & $\begin{array}{l}\text { GCAGAAGATGAGGAAGGTTATCTATTAGA } \\
\text { GCC }\end{array}$ & {$[07,05]$} & $\begin{array}{l}\text { AAAGGAATTAAAACAGAGGTGAGGTGGCT } \\
\text { ATT }\end{array}$ \\
\hline$[06,08]$ & $\begin{array}{l}\text { ATTAACACGGTCAGTTGGCAAATCTTTAGA } \\
\text { AG }\end{array}$ & {$[07,09]$} & $\begin{array}{l}\text { CAATATCTCGCCTGCAACAGTGCCTAAGAA } \\
\text { TA }\end{array}$ \\
\hline$[06,12]$ & $\begin{array}{l}\text { AGCCAGCAAACCTCAAATATCAAACAACTC } \\
\text { GT }\end{array}$ & {$[07,17]$} & $\begin{array}{l}\text { AAAACGACACTCTAGAGGATCCCCGCCCG } \\
\text { CTT }\end{array}$ \\
\hline$[06,20]$ & $\begin{array}{l}\text { AGCTCGAAGGGTTTTCCCAGTCACTCTGG } \\
\text { TGC }\end{array}$ & {$[07,21]$} & $\begin{array}{l}\text { TAACGCCATTCGTAATCATGGTCAAGCTAA } \\
\text { CT }\end{array}$ \\
\hline$[06,24]$ & $\begin{array}{l}\text { TCCTGTGTGTGCTGCAAGGCGATTGCCAT } \\
\text { TCA }\end{array}$ & {$[07,25]$} & $\begin{array}{l}\text { AGGGGGATGAAATTGTTATCCGCTTAAAGT } \\
\text { GT }\end{array}$ \\
\hline$[08,04]$ & $\begin{array}{l}\text { GTCAATAGCTGATTATCAGATGATATTATA } \\
\text { CT }\end{array}$ & {$[09,05]$} & $\begin{array}{l}\text { TCATATTCATAATACATTTGAGGAAACAGTT } \\
\text { G }\end{array}$ \\
\hline$[08,08]$ & $\begin{array}{l}\text { TATTAGACCCACCAGAAGGAGCGGCTACC } \\
\text { ATA }\end{array}$ & {$[09,09]$} & $\begin{array}{l}\text { CAAAGAAATTTACAAACAATTCGACCCTCA } \\
\text { AT }\end{array}$ \\
\hline$[08,12]$ & $\begin{array}{l}\text { ATTAAATCGAGTAACATTATCATTGAAATAA } \\
\text { A }\end{array}$ & {$[09,17]$} & $\begin{array}{l}\text { CGACGACAGCTTTCCGGCACCGCTGACGT } \\
\text { TGT }\end{array}$ \\
\hline$[08,20]$ & $\begin{array}{l}\text { CGGAAACCCGTGCATCTGCCAGTTTAATTC } \\
\text { GC }\end{array}$ & {$[09,21]$} & $\begin{array}{l}\text { ATCGTAACAGGCAAAGCGCCATTCAAGTT } \\
\text { GGG }\end{array}$ \\
\hline$[08,24]$ & $\begin{array}{l}\text { GGCTGCGCGGTCACGTTGGTGTAGTCATC } \\
\text { AAC }\end{array}$ & {$[09,25]$} & $\begin{array}{l}\text { ATGGGATAAACTGTTGGGAAGGGCCTGGC } \\
\text { GAA }\end{array}$ \\
\hline$[10,04]$ & $\begin{array}{l}\text { TCTGAATAAAGTTACAAAATCGCGCAAAAG } \\
\text { AA }\end{array}$ & {$[11,05]$} & $\begin{array}{l}\text { TGAATACCATGGAAGGGTTAGAACAATTAT } \\
\text { CA }\end{array}$ \\
\hline$[10,08]$ & $\begin{array}{l}\text { TCAAAATTAATAACGGATTCGCCTACAAAA } \\
\text { TT }\end{array}$ & {$[11,09]$} & $\begin{array}{l}\text { GGAGAAACATTTGCACGTAAAACATTGCG } \\
\text { GAA }\end{array}$ \\
\hline$[10,12]$ & $\begin{array}{l}\text { GAAATTGCACAGTAACAGTACCTTAATTAC } \\
\text { CT }\end{array}$ & {$[11,17]$} & $\begin{array}{l}\text { AGTGCTGATTCGGACAGAGTGACACATTAA } \\
\text { ATGGAACGCCATCAAAAATGAGGGGA }\end{array}$ \\
\hline$[10,20]$ & $\begin{array}{l}\text { GTCTGGCCACGTTAATATTTTGTTGGTCAT } \\
\text { TG }\end{array}$ & {$[11,21]$} & $\begin{array}{l}\text { AATTGTAATTCCTGTAGCCAGCTTATGGGC } \\
\text { GC }\end{array}$ \\
\hline$[10,24]$ & $\begin{array}{l}\text { ATTAAATGGAAGATTGTATAAGCAGAATCG } \\
\text { AT }\end{array}$ & {$[11,25]$} & $\begin{array}{l}\text { AAAAACAGTGAGCGAGTAACAACCTGACC } \\
\text { GTA }\end{array}$ \\
\hline$[12,04]$ & $\begin{array}{l}\text { GATGATGAGCGATAGCTTAGATTAAAAATC } \\
\text { AT }\end{array}$ & {$[13,05]$} & $\begin{array}{l}\text { AAAACATAAACAAACATCAAGAAAGATTGC } \\
\text { TT }\end{array}$ \\
\hline
\end{tabular}




\begin{tabular}{|c|c|c|c|}
\hline$[12,08]$ & $\begin{array}{l}\text { AATTACATATTAATTTTCCCTTAGCCTCCGG } \\
\text { C }\end{array}$ & {$[13,09]$} & $\begin{array}{l}\text { AGTGCTGATTCGGACAGAGTGACACGCTA } \\
\text { TTATTAACAATTTCATTTGTTACATCG }\end{array}$ \\
\hline$[12,12]$ & $\begin{array}{l}\text { AGTGCTGATTCGGACAGAGTGACATTTTTA } \\
\text { ATAATAACCTTGCTTCTGGTAAATGC }\end{array}$ & {$[13,17]$} & $\begin{array}{l}\text { TGATAAATTCTACAAAGGCTATCAAAAATTC } \\
\text { G }\end{array}$ \\
\hline$[12,20]$ & $\begin{array}{l}\text { AGTGCTGATTCGGACAGAGTGACACCTGA } \\
\text { GAGAATATGATATTCAACCTAATACTT }\end{array}$ & {$[13,21]$} & $\begin{array}{l}\text { TCACCATCTCTGGAGCAAACAAGAAATATT } \\
\text { TA }\end{array}$ \\
\hline$[12,24]$ & $\begin{array}{l}\text { GAACGGTATGAGAAAGGCCGGAGACGCAA } \\
\text { GGA }\end{array}$ & {$[13,25]$} & $\begin{array}{l}\text { CAAAAGGGATCGTAAAACTAGCATAAAGCC } \\
\text { CC }\end{array}$ \\
\hline$[14,04]$ & $\begin{array}{l}\text { AGGTCTGACGTGTGATAAATAAGGTACTAG } \\
\text { AA }\end{array}$ & {$[15,05]$} & $\begin{array}{l}\text { ATACCGACGAGACTACCTTTTTAAAATCCT } \\
\text { TG }\end{array}$ \\
\hline$[14,08]$ & $\begin{array}{l}\text { TTAGGTTGCTGACCTAAATTTAATTTATACA } \\
\text { A }\end{array}$ & {$[15,09]$} & $\begin{array}{l}\text { TTCATCTTGGTTATATAACTATATTAAATCG } \\
\text { T }\end{array}$ \\
\hline$[14,12]$ & $\begin{array}{l}\text { TGATGCAATTTTTCAAATATATTTCTCAACA } \\
\text { G }\end{array}$ & {$[15,17]$} & $\begin{array}{l}\text { AATAAAGCAAACATTATGACCCTGGTTCTA } \\
\text { GC }\end{array}$ \\
\hline$[14,20]$ & $\begin{array}{l}\text { TTGCGGGAGGCAAAGAATTAGCAAGTAGA } \\
\text { TTT }\end{array}$ & {$[15,21]$} & $\begin{array}{l}\text { ACAGGCAAGAAGCCTTTATTTCAACAGTCA } \\
\text { AA }\end{array}$ \\
\hline$[14,24]$ & $\begin{array}{l}\text { TAAAAATTTAGCATTAACATCCAACAAATG } \\
\text { GT }\end{array}$ & {$[15,25]$} & $\begin{array}{l}\text { AATAGTAGTTTAGAACCCTCATATTAAAGAT } \\
\mathrm{T}\end{array}$ \\
\hline$[16,04]$ & $\begin{array}{l}\text { AAAGCCTGAAAGTAATTCTGTCCAGCAGAA } \\
\text { CG }\end{array}$ & {$[17,05]$} & $\begin{array}{l}\text { CAAAAGGTTTTAGTATCATATGCGGGTTTG } \\
\text { AA }\end{array}$ \\
\hline$[16,08]$ & $\begin{array}{l}\text { ATTCTTACTAATAAGAGAATATAAGTCCTGA } \\
\text { A }\end{array}$ & {$[17,09]$} & $\begin{array}{l}\text { AGTGCTGATTCGGACAGAGTGACACGAGC } \\
\text { CAGCAGTATAAAGCCAACGTAGTTAAT }\end{array}$ \\
\hline$[16,12]$ & $\begin{array}{l}\text { TAGGGCTTATGTAATTTAGGCAGAATTTAC } \\
\text { GA }\end{array}$ & {$[17,17]$} & $\begin{array}{l}\text { TACGGTGTCCAATTCTGCGAACGAAATTAA } \\
\text { GC }\end{array}$ \\
\hline$[16,20]$ & $\begin{array}{l}\text { AGTTTGACCATGTTTTTAAATATGCTAATTCG } \\
\text { A }\end{array}$ & {$[17,21]$} & $\begin{array}{l}\text { AGTGCTGATTCGGACAGAGTGACATAGCT } \\
\text { CAACATTAGATACATTTCGTAAATCAT }\end{array}$ \\
\hline$[16,24]$ & $\begin{array}{l}\text { CAATAACCGCTTAATTGCTGAATAGCAAAC } \\
\text { TC }\end{array}$ & {$[17,25]$} & $\begin{array}{l}\text { GGCTTAGATGTTTAGCTATATTTTATTCTAC } \\
\text { T }\end{array}$ \\
\hline$[18,04]$ & $\begin{array}{l}\text { CGCCTGTTTTCATCGTAGGAATCAAGAAGG } \\
\text { СT }\end{array}$ & {$[19,05]$} & $\begin{array}{l}\text { TTTTTATTTATCAACAATAGATAAAGTACCG } \\
\text { A }\end{array}$ \\
\hline$[18,08]$ & $\begin{array}{l}\text { CAAGAAAACACTCATCGAGAACAAGCGTTT } \\
\text { TA }\end{array}$ & {$[19,09]$} & $\begin{array}{l}\text { AGTGCTGATTCGGACAGAGTGACAAAGTA } \\
\text { CCGATAATATCCCATCCTAGGCATTTT }\end{array}$ \\
\hline$[18,12]$ & $\begin{array}{l}\text { GCATGTAGCATTCCAAGAACGGGTTTTTGA } \\
\text { AG }\end{array}$ & {$[19,17]$} & $\begin{array}{l}\text { GCGGATTGTTCAAATATCGCGTTTAACTAA } \\
\text { AG }\end{array}$ \\
\hline$[18,20]$ & $\begin{array}{l}\text { GCTTCAAACTGACTATTATAGTCAGCCAGA } \\
\text { GG }\end{array}$ & {$[19,21]$} & $\begin{array}{l}\text { TCTTTACCGCGAACCAGACCGGAATAATG } \\
\text { CTG }\end{array}$ \\
\hline$[18,24]$ & $\begin{array}{l}\text { CAACAGGTAATGACCATAAATCAAGGATAG } \\
\text { CG }\end{array}$ & {$[19,25]$} & $\begin{array}{l}\text { AAAACGAGCAGGATTAGAGAGTACTTGCG } \\
\text { GAT }\end{array}$ \\
\hline$[20,04]$ & $\begin{array}{l}\text { TATCCGGTAATAAACAGCCATATTTGTTTAAA } \\
\text { C }\end{array}$ & {$[21,05]$} & $\begin{array}{l}\text { AGTTACAAATTCTAAGAACGCGAGGCAAG } \\
\text { CCG }\end{array}$ \\
\hline$[20,08]$ & $\begin{array}{l}\text { GCGAACCTCGTCTTTCCAGAGCCTTACAG } \\
\text { AGA }\end{array}$ & {$[21,09]$} & $\begin{array}{l}\text { CTAACGAGCCCGACTTGCGGGAGGATTAA } \\
\text { ACC }\end{array}$ \\
\hline
\end{tabular}




\begin{tabular}{|c|c|c|c|}
\hline$[20,12]$ & $\begin{array}{l}\text { CCTTAAATATTTTATCCTGAATCTCATTAGA } \\
\text { C }\end{array}$ & {$[21,17]$} & $\begin{array}{l}\text { TTTACCAGTTGCAAAAGAAGTTTTGAAGCA } \\
\text { AA }\end{array}$ \\
\hline$[20,20]$ & $\begin{array}{l}\text { AGTGCTGATTCGGACAGAGTGACAGGGTA } \\
\text { ATAAGAGCAACACTATCATCGTTGGGA }\end{array}$ & {$[21,21]$} & $\begin{array}{l}\text { GCATAGTAGTAAAATGTTTAGACTAAATCA } \\
\text { GG }\end{array}$ \\
\hline$[20,24]$ & $\begin{array}{l}\text { TCCAATACCATAACGCCAAAAGGAAACTAA } \\
\text { CG }\end{array}$ & {$[21,25]$} & $\begin{array}{l}\text { TGCAGATATGCGGAATCGTCATAACAGTTC } \\
\text { AG }\end{array}$ \\
\hline$[22,04]$ & $\begin{array}{l}\text { GTCAAAAAAAACAATGAAATAGCAAGTAAG } \\
\text { CA }\end{array}$ & {$[23,05]$} & $\begin{array}{l}\text { AGAGCAAGTGAAAATAGCAGCCTTAATTTG } \\
\text { CC }\end{array}$ \\
\hline$[22,08]$ & $\begin{array}{l}\text { GAATAACAAGAATTGAGTTAAGCCGGAAAC } \\
\text { CG }\end{array}$ & {$[23,09]$} & $\begin{array}{l}\text { AACCCACATAAAAACAGGGAAGCGTACCA } \\
\text { ACG }\end{array}$ \\
\hline$[22,12]$ & $\begin{array}{l}\text { AGTGCTGATTCGGACAGAGTGACAGGGAG } \\
\text { AATATTGAGCGCTAATATCCCCAAAAG }\end{array}$ & {$[23,17]$} & $\begin{array}{l}\text { AGTGCTGATTCGGACAGAGTGACAATCATT } \\
\text { GTATTATACCAGTCAGGAAACCCTCG }\end{array}$ \\
\hline$[22,20]$ & $\begin{array}{l}\text { AGAAAAATTGAGATGGTTTAATTTGGCGCA } \\
\text { TA }\end{array}$ & {$[23,21]$} & $\begin{array}{l}\text { ATTGGGCTCTACGTTAATAAAACGATTACG } \\
\text { AG }\end{array}$ \\
\hline$[22,24]$ & $\begin{array}{l}\text { GAACAACAGAGAAACACCAGAACGAATCTT } \\
\text { GA }\end{array}$ & {$[23,25]$} & $\begin{array}{l}\text { GCCCTGACTTATTACAGGTAGAAATCAACT } \\
\text { AA }\end{array}$ \\
\hline$[24,04]$ & $\begin{array}{l}\text { GATAGCCGTAAGTTTATTTTGTCAGCCAAA } \\
\text { GA }\end{array}$ & {$[25,05]$} & $\begin{array}{l}\text { CCACGGAAAACAAAGTTACCAGAACAATAA } \\
\text { TA }\end{array}$ \\
\hline$[24,08]$ & $\begin{array}{l}\text { AGGAAACGACATATAAAAGAAACGGAGGG } \\
\text { AGG }\end{array}$ & {$[25,09]$} & $\begin{array}{l}\text { AGGTGGCACAATAATAACGGAATAAGAGA } \\
\text { GAT }\end{array}$ \\
\hline$[24,12]$ & $\begin{array}{l}\text { AACTGGCACAAACGTAGAAAATACTTCATT } \\
\text { AA }\end{array}$ & {$[25,17]$} & $\begin{array}{l}\text { TAAGGGAAAACGGTGTACAGACCACAACT } \\
\text { TTA }\end{array}$ \\
\hline$[24,20]$ & $\begin{array}{l}\text { GGCTGGCTGAACGAGGCGCAGACGCGAA } \\
\text { AGAG }\end{array}$ & {$[25,21]$} & $\begin{array}{l}\text { CTTAGCCGGACCTTCATCAAGAGTAGTAGT } \\
\text { AA }\end{array}$ \\
\hline$[24,24]$ & $\begin{array}{l}\text { CAAGAACCAAATCCGCGACCTGCTATCTTT } \\
\text { GA }\end{array}$ & {$[25,25]$} & $\begin{array}{l}\text { TTGTGTCGGGATATTCATTACCCATAAGGC } \\
\text { TT }\end{array}$ \\
\hline$[26,04]$ & $\begin{array}{l}\text { CAAAAGGGAACCATCGATAGCAGCCTTTA } \\
\text { GCG }\end{array}$ & {$[27,05]$} & $\begin{array}{l}\text { ACCAATGACGACATTCAACCGATTCAAAGA } \\
\text { CA }\end{array}$ \\
\hline$[26,08]$ & $\begin{array}{l}\text { GAAGGTAAACCATTAGCAAGGCCGGCATT } \\
\text { TTC }\end{array}$ & {$[27,09]$} & $\begin{array}{l}\text { GCACCATTATATTGACGGAAATTAATACAT } \\
\text { AA }\end{array}$ \\
\hline$[26,12]$ & $\begin{array}{l}\text { AGGTGAATTTAGAGCCAGCAAAATTGCCAT } \\
\text { CT }\end{array}$ & {$[27,17]$} & $\begin{array}{l}\text { AGTTTCCAAGGCACCAACCTAAAAGTCAAT } \\
\text { CA }\end{array}$ \\
\hline$[26,20]$ & $\begin{array}{l}\text { GCAAAAGAGGACTAAAGACTTTTTTGACAA } \\
\text { CA }\end{array}$ & {$[27,21]$} & $\begin{array}{l}\text { GGCTTTGAATACACTAAAACACTCCCATGT } \\
\text { TA }\end{array}$ \\
\hline$[26,24]$ & $\begin{array}{l}\text { CCCCCAGCAACGAGGGTAGCAACGTATTC } \\
\text { GGT }\end{array}$ & {$[27,25]$} & $\begin{array}{l}\text { AGCATCGGGATTATACCAAGCGCGCTGAT } \\
\text { AAA }\end{array}$ \\
\hline$[28,04]$ & $\begin{array}{l}\text { TCAGACTGCCACCAGAACCACCACGGCAG } \\
\text { GTC }\end{array}$ & {$[29,05]$} & $\begin{array}{l}\text { CAGAGCCGTAGCGCGTTTTCATCGGAAAC } \\
\text { GTC }\end{array}$ \\
\hline$[28,08]$ & $\begin{array}{l}\text { GGTCATAGCGCCACCCTCAGAGCCAACAA } \\
\text { ATA }\end{array}$ & {$[29,09]$} & $\begin{array}{l}\text { CTCAGAACCCCCCTTATTAGCGTTCACCAG } \\
\text { TA }\end{array}$ \\
\hline$[28,12]$ & $\begin{array}{l}\text { TTTCATAAACCGCCTCCCTCAGAGAAGCG } \\
\text { CAG }\end{array}$ & {$[29,17]$} & $\begin{array}{l}\text { GCTTGCTTATAGTTGCGCCGACAACATGA } \\
\text { GGA }\end{array}$ \\
\hline
\end{tabular}




\begin{tabular}{|c|c|c|c|}
\hline$[28,20]$ & $\begin{array}{l}\text { ACCATCGCGCCTTTAATTGTATCGTTAGTA } \\
\text { AA }\end{array}$ & {$[29,21]$} & $\begin{array}{l}\text { CAAAAGGACCACGCATAACCGATAGCTAC } \\
\text { AGA }\end{array}$ \\
\hline$[28,24]$ & $\begin{array}{l}\text { CGCTGAGGTGAAAATCTCCAAAAATAAACA } \\
\text { AC }\end{array}$ & {$[29,25]$} & $\begin{array}{l}\text { TTTCACGTCTTGCAGGGAGTTAAACGAAAG } \\
\text { AC }\end{array}$ \\
\hline$[30,04]$ & $\begin{array}{l}\text { AGACGATTCGTATAAACAGTTAATAAACAT } \\
\text { GA }\end{array}$ & {$[31,05]$} & $\begin{array}{l}\text { ACAGTGCCGGCCTTGATATTCACAACCAC } \\
\text { СCT }\end{array}$ \\
\hline$[30,08]$ & $\begin{array}{l}\text { AATCCTCATTAACGGGGTCAGTGCCAAGA } \\
\text { GAA }\end{array}$ & {$[31,09]$} & $\begin{array}{l}\text { AATAAGTTTTAAAGCCAGAATGGACCGCCA } \\
\text { CC }\end{array}$ \\
\hline$[30,12]$ & $\begin{array}{l}\text { TCTCTGAATTGATGATACAGGAGTTCAGTA } \\
\text { CC }\end{array}$ & {$[31,17]$} & $\begin{array}{l}\text { ACAGACAGGTCGTCTTTCCAGACGGTTTAT } \\
\text { CA }\end{array}$ \\
\hline$[30,20]$ & $\begin{array}{l}\text { TGAATTTTAAACTACAACGCCTGTCACCGT } \\
\text { AC }\end{array}$ & {$[31,21]$} & $\begin{array}{l}\text { ACCAGTACCTGTATGGGATTTTGCAAAGGC } \\
\text { TC }\end{array}$ \\
\hline$[30,24]$ & $\begin{array}{l}\text { TTTCAACATGTACCGTAACACTGATCAGAA } \\
\text { CC }\end{array}$ & {$[31,25]$} & $\begin{array}{l}\text { GGAACCCAGTTTCAGCGGAGTGAGAATAA } \\
\text { TTT }\end{array}$ \\
\hline \multicolumn{4}{|c|}{ Red staples } \\
\hline Name & Sequence & Name & Sequence \\
\hline$[02,16]$ & $\begin{array}{l}\text { GATAGGGTTTATAAATCAAAAGAAGTAGCA } \\
\text { AT }\end{array}$ & {$[17,13]$} & $\begin{array}{l}\text { ACGCCAACAATTGAGAATCGCCATATATAA } \\
\text { CA }\end{array}$ \\
\hline$[03,13]$ & $\begin{array}{l}\text { ACTTCTTTACGCAAATTAACCGTTTAGCCC } \\
\text { GA }\end{array}$ & {$[18,16]$} & $\begin{array}{l}\text { CGAAAGACCATCAAAAAGATTAAGGGCTGT } \\
\text { CT }\end{array}$ \\
\hline$[04,16]$ & $\begin{array}{l}\text { TAATGAATGGAAACCTGTCGTGCCAACAGA } \\
\text { GA }\end{array}$ & {$[19,13]$} & $\begin{array}{l}\text { TTCCTTATAAACCAATCAATAATCAGGAAG } \\
\text { CC }\end{array}$ \\
\hline$[05,13]$ & $\begin{array}{l}\text { TAGAACCCAAGGGACATTCTGGCCAGCTG } \\
\text { CAT }\end{array}$ & {$[20,16]$} & $\begin{array}{l}\text { AGAGGCTTACGACGATAAAAACCATTTGCA } \\
\text { CC }\end{array}$ \\
\hline$[06,16]$ & $\begin{array}{l}\text { GCAGGTCGGGCCAGTGCCAAGCTTAAGCA } \\
\text { TCA }\end{array}$ & {$[21,13]$} & $\begin{array}{l}\text { CAGCTACACAAGATTAGTTGCTATAAATAG } \\
\text { CG }\end{array}$ \\
\hline$[07,13]$ & $\begin{array}{l}\text { CCTTGCTGGCAAATGAAAAATCTAGCATGC } \\
\text { СT }\end{array}$ & {$[22,16]$} & $\begin{array}{l}\text { ACTGGCTCGAATTACCTTATGCGAACAAAG } \\
\text { TC }\end{array}$ \\
\hline$[08,16]$ & $\begin{array}{l}\text { TCCAGCCAGTATCGGCCTCAGGAATTAATT } \\
\text { TT }\end{array}$ & {$[23,13]$} & $\begin{array}{l}\text { AGAGGGTATAACTGAACACCCTGATTTTAA } \\
\text { GA }\end{array}$ \\
\hline$[09,13]$ & $\begin{array}{l}\text { AAAAGTTTCTTTGCCCGAACGTTAGATCGC } \\
\text { AC }\end{array}$ & {$[24,16]$} & $\begin{array}{l}\text { GACAGATGCCGAACTGACCAACTTTTACG } \\
\text { CAG }\end{array}$ \\
\hline$[10,16]$ & $\begin{array}{l}\text { AACCAATATTTTGTTAAATCAGCTAACGTCA } \\
\text { G }\end{array}$ & {$[25,13]$} & $\begin{array}{l}\text { TATGTTAGTGATTAAGACTCCTTATGAAAG } \\
\text { AG }\end{array}$ \\
\hline$[11,13]$ & $\begin{array}{l}\text { ATGAATATGTAGATTTTCAGGTTTCATTTTT } \\
\mathrm{T}\end{array}$ & {$[26,16]$} & $\begin{array}{l}\text { CACTACGATTAAACGGGTAAAATATTGAGC } \\
\text { CA }\end{array}$ \\
\hline$[12,16]$ & $\begin{array}{l}\text { TTGAGAGATAATGCCGGAGAGGGTTCAAT } \\
\text { ATA }\end{array}$ & {$[27,13]$} & $\begin{array}{l}\text { TTTGGGAATATCACCGTCACCGACCGTAAT } \\
\text { GC }\end{array}$ \\
\hline$[13,13]$ & $\begin{array}{l}\text { TGTGAGTGGGAAACAGTACATAAAAGCTAT } \\
\text { TT }\end{array}$ & {$[28,16]$} & $\begin{array}{l}\text { TGATACCGTCGAGGTGAATTTCTTCAGAGC } \\
\text { CA }\end{array}$ \\
\hline$[14,16]$ & $\begin{array}{l}\text { TGTACCAACTCAGAGCATAAAGCTAAGAAC } \\
\text { GC }\end{array}$ & {$[29,13]$} & $\begin{array}{l}\text { CCACCGGATCAAAATCACCGGAACAAACA } \\
\text { GCT }\end{array}$ \\
\hline
\end{tabular}




\begin{tabular}{|l|l|l|l|}
{$[15,13]$} & $\begin{array}{l}\text { GAGAAAACATCCAATCGCAAGACAAAATCG } \\
\text { GT }\end{array}$ & {$[30,16]$} & $\begin{array}{l}\text { AAAGTTTTCCCTCATAGTTAGCGTGCGTCA } \\
\text { TA }\end{array}$ \\
\hline$[16,16]$ & $\begin{array}{l}\text { GTTGATTCCTGGAAGTTTCATTCCATTTAA } \\
\text { CA }\end{array}$ & {$[31,13]$} & $\begin{array}{l}\text { CATGGCTTTTTACCGTTCCAGTAAAACGAT } \\
\text { CT }\end{array}$ \\
\hline
\end{tabular}




\section{Characterization}

\section{AFM images of tubular and rectangular DNA origami}

AFM imaging were performed to examine the correct capping between caps and pores as designed in Figure S1. We conjugated origami caps with DNA pores with a molar ratio of 2:1 to ensure as many origami pores capped as possible. For deposition of samples for AFM imaging, the target sample was diluted to $1.0 \mathrm{nM}$ with TAEM buffer. Then, an aliquot of $10 \mu \mathrm{L}$ diluted sample was pipetted onto mica surface and incubated for 5 minutes at room temperature. After incubation, the mica was blown dry with compressed air, rinsed with $80 \mu \mathrm{L}$ deionized (DI) water for about 3 seconds, and then blown dry again with compressed air.

AFM imaging was performed in air using the Peak-Force tapping mode with a Bruker Dimension Icon AFM and SCANASYST-AIR probes. The images show that more than $90 \%$ of the examined pores are connected with flat rectangular caps (Figure S2c and d). The binding yield was obtained by counting the number of bound cap-pore structures in the AFM images (and height profiles) and compared them to the number of unbound origami structures (Figure S2). The connection is also confirmed by the height profiles (Figure S2e) as the height of cap (blue) and pore (red) add up to approximately the height of capped pore (pink). After the cap releasers were incubated for 1 hour, nearly $90 \%$ of the pores are detached from the flat origami (Figure S2f), confirming that the cap releasers are effective in removing the caps and opening the origami pores. The yield refers to the percentage of origami pores originally bound with caps and switched to the status of unbound with caps. 

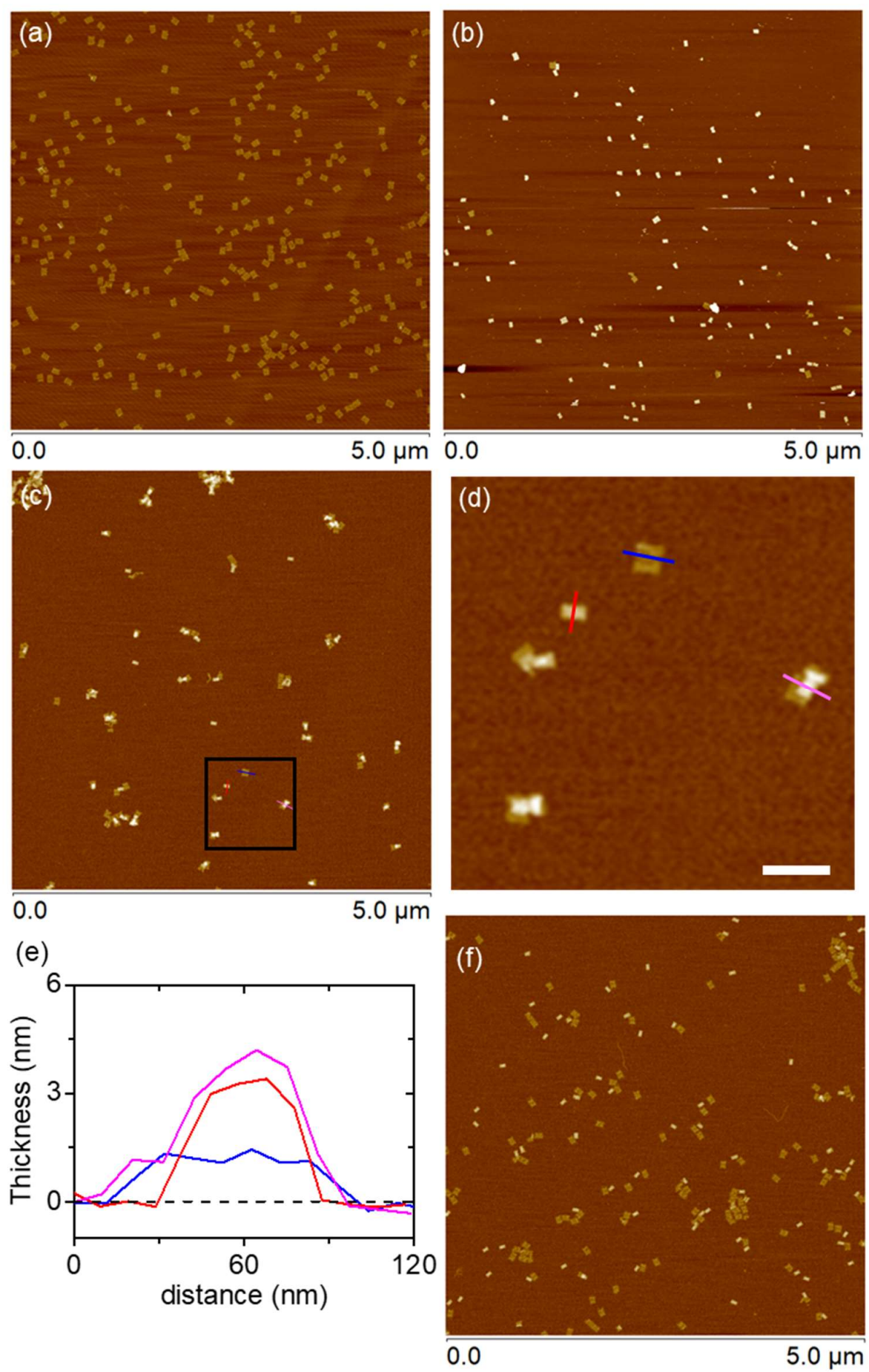

Figure S2. AFM images of DNA origami. (a) Near 100\% flat origami caps, which measure approximately $60 \mathrm{~nm} \times 100 \mathrm{~nm}$ in dimension. (b) The majority of DNA origami measures approximately $60 \mathrm{~nm} \times 50 \mathrm{~nm}$ with a thickness of $\sim 4 \mathrm{~nm}$, which is roughly half the size and twice the thickness of the origami cap. The results indicate the identity of the tubular structure of the origami in solution, which should have a diameter of $\sim 32 \mathrm{~nm}$ and a length of $\sim 60 \mathrm{~nm}$. Note that the tubular structures collapsed into a rectangular shape to maximize their contact with the mica substrate. (c) Origami structures after connecting caps with pores at a ratio of 2: 
1. Most pores are connected to caps, while the linked origami structures in $3 \mathrm{D}$ collapsed before or during the AFM imaging. (d) Zoom-in of the black square area in (c). The scale bar is 20 $\mathrm{nm}$. Colored lines (blue, red, and pink) denote the sites where heights are measured. (e) Corresponding height profiles of the objects in (d). Cross-sections of a cap (blue), an isolated pore (red), and a pore connected with a cap (pink). Dashed line indicates the mica substrate. The profiles confirm the connection as the height of cap and pore add up to approximately the height of capped pore. (f) Origami caps and pores after mixing with cap releaser strands. The majority $(\sim 90 \%)$ of the pores can be observed as separated from the caps, suggesting that cap releasers are effective in removing the flat caps from the pores.

\section{Imaging system setup}

A custom-built inverted fluorescence microscope (Zeiss Axio Observer D1) was used for imaging. Three diode lasers at 405, 561, and $658 \mathrm{~nm}$ (Laserglow) were used as light source. An oil-immersion $63 \times$ objective lens from Zeiss was used, and the collected emission light from the sample was imaged with an Andor iXon3 electron multiplying charge coupled device (EMCCD) camera. Exemplary images are shown in Figure. The images showed full-scale images $(135 \mu \mathrm{m} \times 135 \mu \mathrm{m})$ of a GUV containing Cy5-DNA under bright field and epifluorescence ( $658 \mathrm{~nm}$ laser). A GUV can be clearly seen in both images of bright field and epifluorescence.

(a)

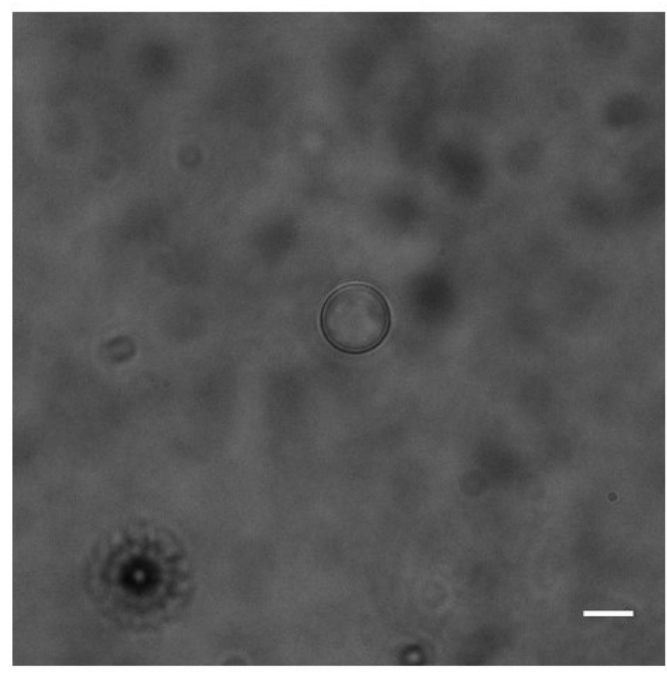

(b)

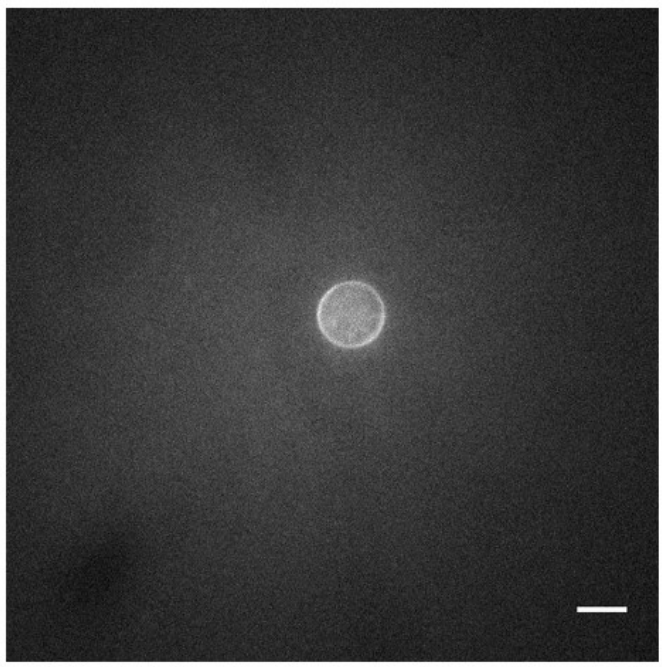

Figure S3. Full-scale images of a GUV containing Cy5-DNA. Scale bars are $10 \mu \mathrm{m}$. (a) Image taken under bright field. (b) Image taken with epi-fluorescence with $658 \mathrm{~nm}$ laser excitation.

\section{Förster resonance energy transfer (FRET) measurement of cap-pore binding}

FRET experiment is commonly used to demonstrate binding between oligonucleotides. ${ }^{3-4}$ In our experiment, we chose the FAM and TAMRA as the FRET pair to confirm correct binding between the DNA origami cap and pore. FAM is a fluorescein which has an emission spectrum overlaps with the absorption spectrum of TAMRA; thus, when FAM and TAMRA are separate, strong fluorescence will be observed from FAM. In contrast, the fluorescence emission will be quenched when FAM and TAMRA are in close proximity due to the energy transfer by dipoledipole coupling. 
We modified the $[21,09]$ staple with FAM at 3' end (CTA ACG AGC CCG ACT TGC GGG AGG ATT AAA CC/36-FAM/) in the DNA origami cap, shown in green in the schematic of Figure S4a. Rest of the staples remain the same as listed in Table S2. Then, TAMRA modified (5' end) short strands (/56-TAMN/TGTCACTCTG) were introduced (shown in red in Figure S4b). This 10-nt sequence is complementary to the green staple with FAM. Thus, the green and red strands base-pair each other, which put FAM and TAMRA in close proximity and quenches the FAM fluorescein. We then added the pore origami which has a 16-nt domain complementary to the green staple. With 16-bp complementarity, the pore replaced the TAMRA-modified strand via toehold-mediated strand displacement and bound to the cap origami (Figure S4c). This even resulted in the recovery of FAM fluorescence.

Figure S4d shows that initially the FAM fluorescence is observed from the solution containing $100 \mu \mathrm{L} 10 \mathrm{nM}$ origami cap with FAM modified staples (green line). The addition of $1 \mu \mathrm{L} 1 \mathrm{mM}$ TAMRA modified strands leads to the binding of TAMRA-strands on the cap, resulting in a fluorescence quenching (blue line). The introduction of $100 \mu \mathrm{L} 10 \mathrm{nM}$ tubular pore origami results in the release of the TAMRA strands and recovery of FAM fluorescence (yellow line). The results confirm the binding between the caps and pores.

Control experiments were also performed. Figure S4e and S4f show that buffer solution and the origami pore without green strands do not result in no apparent signal recovery. The control experiments further verify our design of tubular pores and rectangular caps and their bindings.

(a)

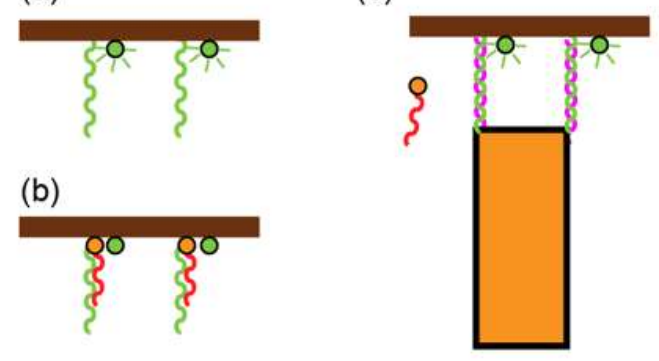

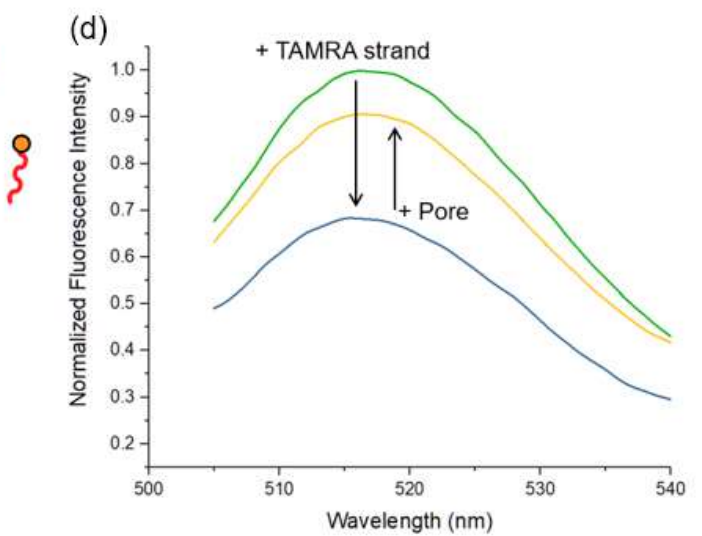

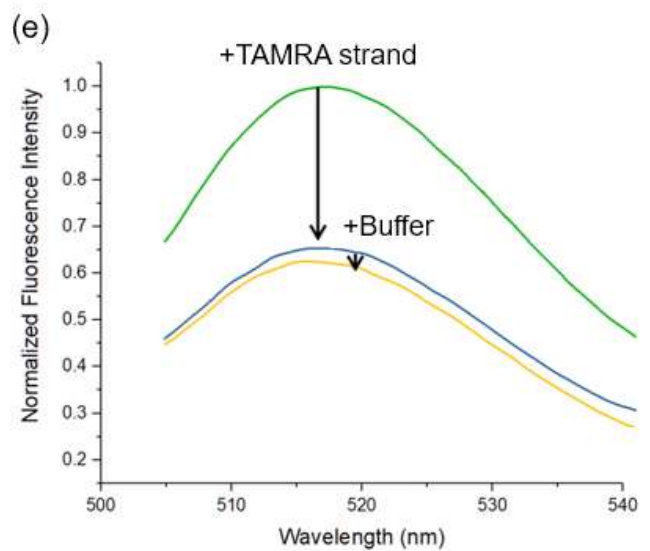

(f)

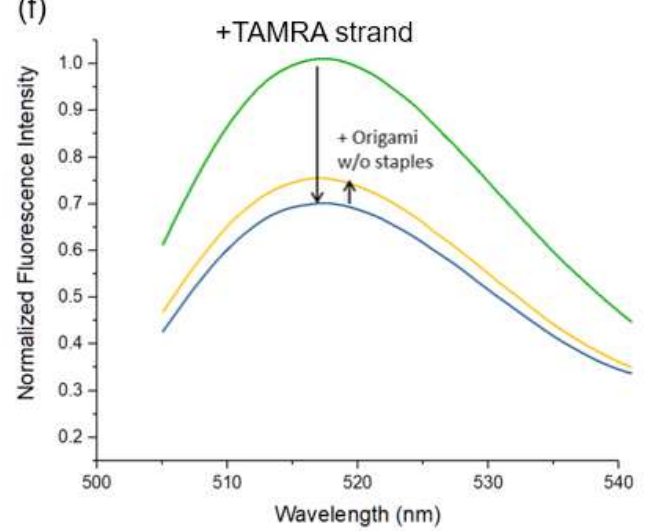


Figure S4. FRET measurements for DNA origami cap-pore binding. (a) FAM fluorescein (green dot) is attached on the cap origami. (b) The short TAMRA strand (red) binds to the staples on the cap and quenches the FAM. (c) When the cap binds with the origami pore (also see Figure S1g), the pore will replace the short TAMRA strand, resulting in the recovery of FAM fluorescence. (d) Green line shows the emission spectrum of the cap origami with FAM modified staples, as illustrated in (a). After addition of short TAMRA strands, the fluorescence drops significantly (blue line) due to FAM-TAMRA quenching with hybridization (illustrated in (b)). When the pore origami is added and linked to the cap origami, the short TAMRA strands are replaced, with fluorescence recovered due to the separation of FAM and TAMRA (yellow), as illustrated in (c). (e) Control experiment with buffer solution. A fluorescence drop is observed (green to blue) with the TAMRA strands. However, there is no fluorescence recovery with buffer solution added (yellow). (f) Control experiment with DNA origami sheets without staples listed in Table S1 (thus, only M13mp18 scaffold). A fluorescence drop is observed after TAMRA strand addition (green to blue). However, no significant change is observed after addition of the DNA origami sheets without staples (yellow).

\section{Additional kinetic measurement}

To measure the kinetics of molecular transport via origami channel, we used GFP and Cy5DNA in the outflow experiment (Figure 3 and Figure S5). GFP and Cy5-DNA were initially encapsulated inside the giant vesicles at a concentration of $\sim 2 \mu \mathrm{M}$. The origami channels were initially closed with rectangular caps. The fluorescence intensity did not change without adding cap releaser strands to open the origami pores, indicating no significant leakage of fluorescent molecules from the GUV. After $\sim 25$ mins of the observation, we added the cap releasers as indicated by black arrows in Figure S5b and S5f. Shortly after that (roughly 5 mins), the fluorescence intensity inside the GUV started to drop. The intensity continued to decrease over time. However, the intensity inside the GUV was still higher than the background after 90 mins. In the control experiments (Figure S5c, S5d, S5g, S5h), the giant vesicles contained the fluorescent molecules, but did not have origami pores embedded on surfaces. As expected, no significant changes in the fluorescence intensity were observed, indicating that there will be no molecular diffusion in and out of the vesicle without origami channels.

To obtain the statistics of kinetic measurements, the experiments were repeated multiple times. To compare different vesicles, we normalized the fluorescence intensities from measurements and calculated the average fluorescence intensity before and after the addition of cap releaser strands. Our results show that for the fluorescence intensity of Cy5-DNA molecules drops from $100 \%$ to approximately $46 \%$ (Figure S6a) and the GFP signal decreases to about $57 \%$ (Figure $\mathrm{S6b})$ on average. Both cases demonstrate significant decreases in fluorescence signals, confirming the release of the origami caps from the GUVs and outflux of fluorescent molecules through the membrane channels.

To better understand the kinetics of molecular outflux, we used a single exponential function $I(t)=a e^{-k_{f} t}+b$ for curve-fitting fluorescence intensity $I(t)$ as a function of time $t$. Here, $k_{f}$ is the molecule transportation rates, and $b$ represents the energy barrier that inhibits further translocation of fluorescent molecules via origami channels. We used multiple datasets and 
calculated the average time constants of GFP and Cy5-DNA:

$$
\begin{gathered}
k_{f-G F P}=0.14 \pm 0.11 \\
k_{f-C y 5}=0.070 \pm 0.04
\end{gathered}
$$

The kinetics of dFITC through a vesicle via origami pores in a previous report by Thomsen et al. ${ }^{5}$ is expressed as:

$$
k_{f-d F I T C}=0.06495
$$

The three transportation rates are similar within the order of magnitude $\left(k_{f, G F P}>k_{f, C y 5-D N A} \approx\right.$ $\left.k_{f, d F I T C}\right)$ and the time constants are about 7,15 , and 15 mins for GFP, Cy5-DNA, and dFITC, respectively.
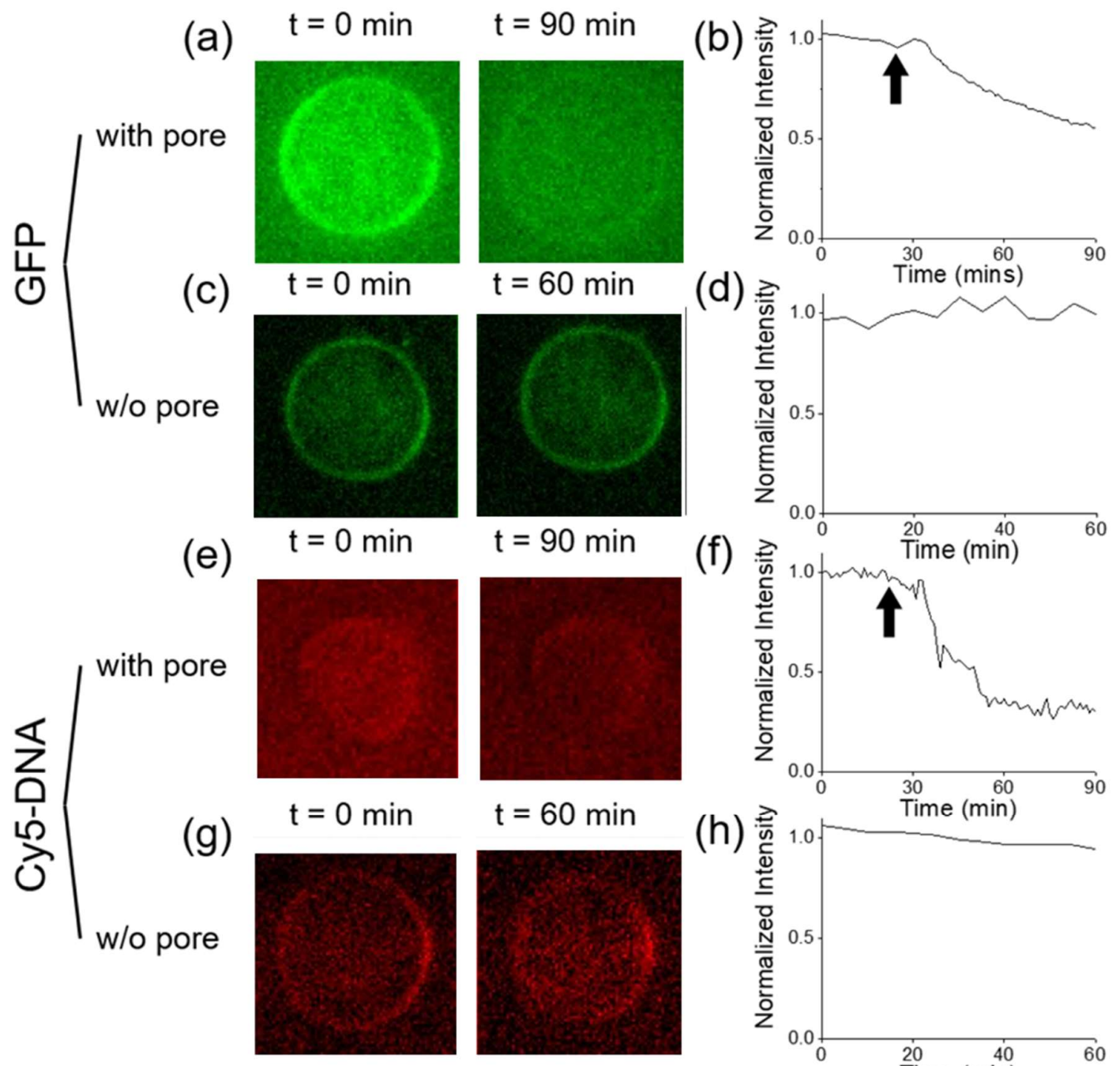

(f)

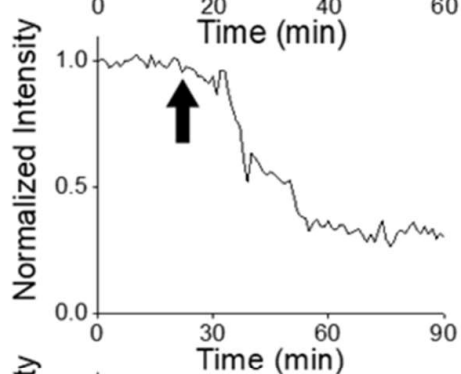

(h)

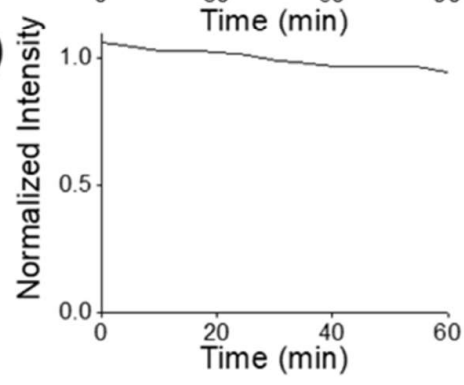

Figure S5. Kinetic measurements of molecular transport through DNA origami pores. (a) Fluorescence images of GFP molecules encapsulated inside a GUV with origami pores at the beginning ( $t=0 \mathrm{~min}$ ) and end ( $t=90 \mathrm{~min}$ ) of the experiment. (b) Normalized fluorescence intensity of GFP as a function of time. After 25 mins, the cap releaser strands were introduced to the imaging chamber to remove the origami caps and open the channels, as indicated by the black arrow. Shortly after that, the fluorescent molecules diffused out of the GUV, and the 
fluorescence intensity inside GUV dropped. (c)-(d) Control experiment with the GUV without pores encapsulating GFP. The fluorescence intensity does not change significantly, indicating that no molecular transportation in and out of the vesicle without pores. (e)-(f) Fluorescence measurement of Cy5-DNA encapsulated inside a giant vesicle over time. Fluorescence images at the beginning and end of the experiment ( $t=0$ and $90 \mathrm{~min}$, respectively). Shortly after adding cap releaser strands (black arrow), the fluorescence intensity inside the GUV dropped. (g)-(h) Control experiment with the GUV encapsulating Cy5-DNA without origami channels. The fluorescence intensity remains constant over time in the absence of origami pores.

(a)

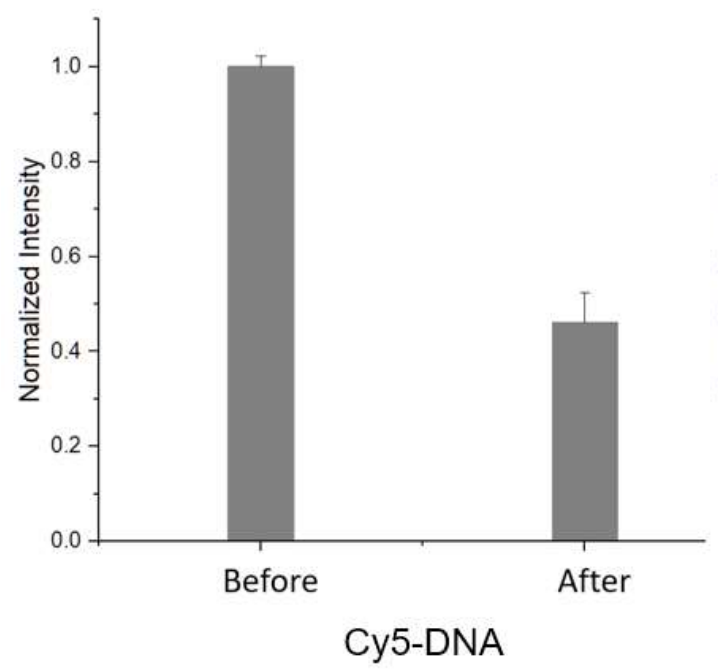

(b)

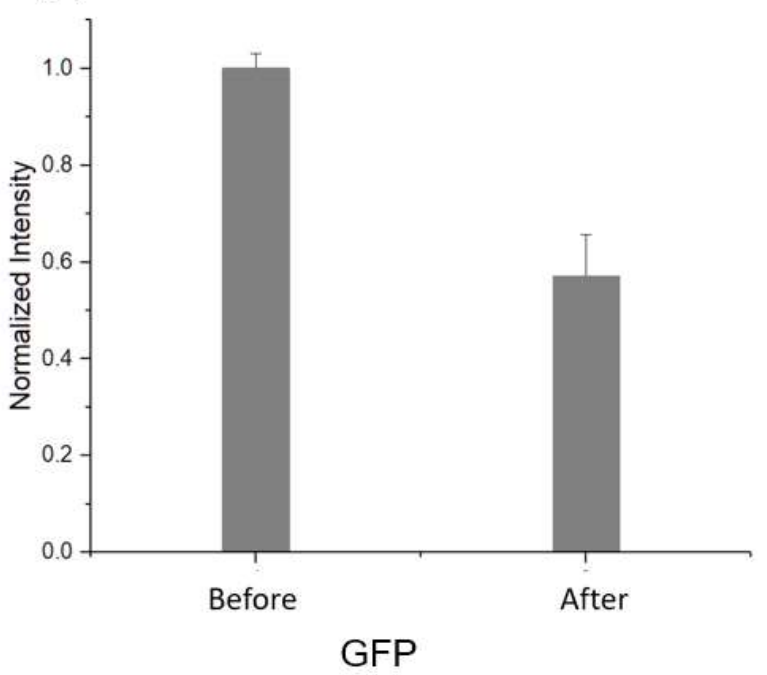

Figure S6. Average fluorescence intensity before and after addition of cap-releaser strands. (a) Normalized fluorescence intensity of GUVs containing Cy5-DNA decreases from $100 \%$ to $\sim 46 \%$ after addition of cap-releaser strands (b) Normalized fluorescence intensity of GUVs with GFP decreased from $100 \%$ to $\sim 57 \%$ after addition of cap-releaser strands.

\section{Exo III activity inside a giant vesicle}

In our experiment, Exo III enzymes are used to transduce external DNA signals to another form of signals inside a giant vesicle. The hairpin strands that enter the GUV are partly digested by the enzyme, exposing the SUV linker domain. The enzymes are functionalized on the surface of polystyrene particles with a diameter of $\sim 200 \mathrm{~nm}$. Restricted by the particle, the enzymes will not pass through the origami channels that have a diameter of about $32 \mathrm{~nm}$. To prove that Exo III is functional inside the giant vesicle, we examined cleavage activity of Exo III in free solution and particle-enzymes inside a GUV using the fluorophore-quencher pair. We used a FAM modified strand (termed FAM-DNA) and its complementary strand with a quencher group (TAMRA-cDNA). When hybridized, the TAMRA will quench the fluorescence of the FAM-DNA via energy transfer, resulting in a fluorescence drop. For testing the free Exo III enzymes, we first prepared a $1 \mu \mathrm{M}$ FAM-DNA solution which has a high fluorescence intensity initially as shown in Figure S7a. Then, we added the solution of TAMRA-cDNA at a ratio of 1:1, and the fluorescence intensity immediately dropped. Next, we added $1 \mathrm{nM}$ Exo III 
that will digest the TAMRA-cDNA. The fluorescence intensity increased (Figure S7a), indicating that Exo III cleaved off TAMRA-cDNA and FAM-DNA is thus released.

We also examined the activity of the particle-modified Exo III enzymes and repeat the previous experiment. Initially, the solution only demonstrates background fluorescence intensity. Then we observed a sharp increase of the fluorescence intensity after adding enzyme-particles to the FAM-DNA/TAMRA-cDNA solution, as shown in Figure S7b. Next, we encapsulated the particle-Exo III into the giant vesicles with open origami pores. This time, the fluorescence intensity increase was small, yet distinct upon addition of GUVs containing enzyme-particles (Figure S7c and S7d). We note that the degree of fluorescence recovery upon addition of Exo III varies significantly. For example, the fluorescence recovery with particle-modified enzymes is much less than that with free enzymes, and it is even smaller with particle-enzymes inside giant vesicles. This may be attributed to the fact that the amount of enzymes was reduced significantly during the processes of conjugation, assembly, encapsulation, and purification. Further, the diffusion of oligonucleotides into and out of vesicles through origami channels may present another barrier. Nonetheless, our experiment clearly demonstrates that enzymes are required for the cleavage of DNA signals. The signals can be received, transduced, and transmitted by synthetic cells, which is used to program reversible aggregation behaviors.

(a)

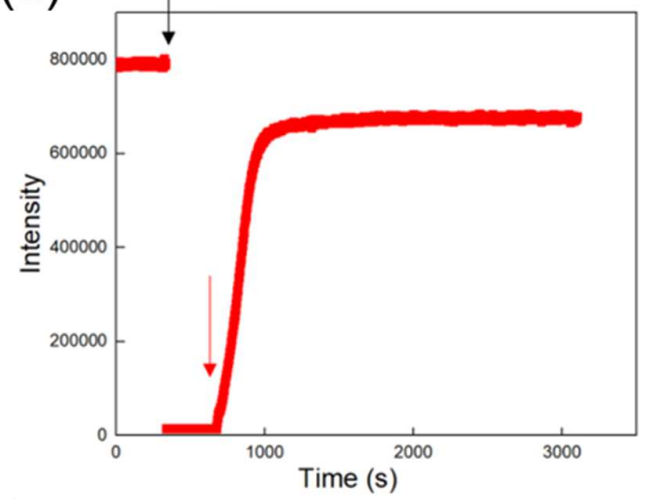

(c)

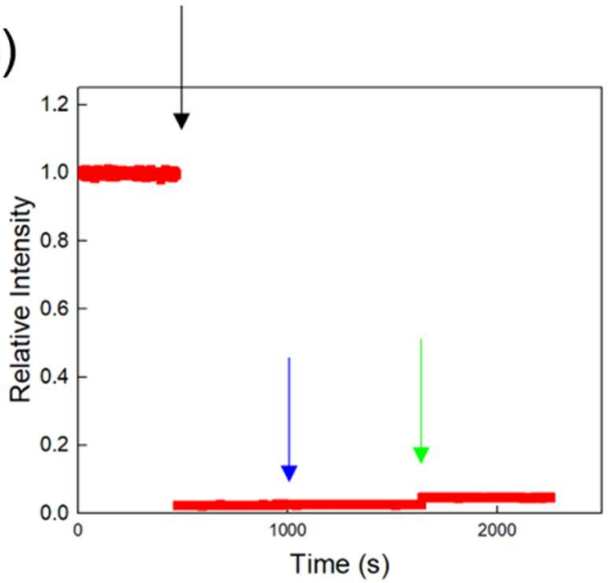

(b)

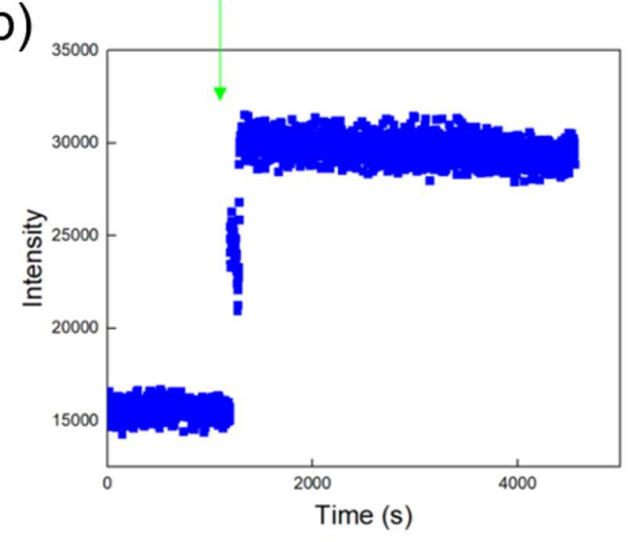

(d)

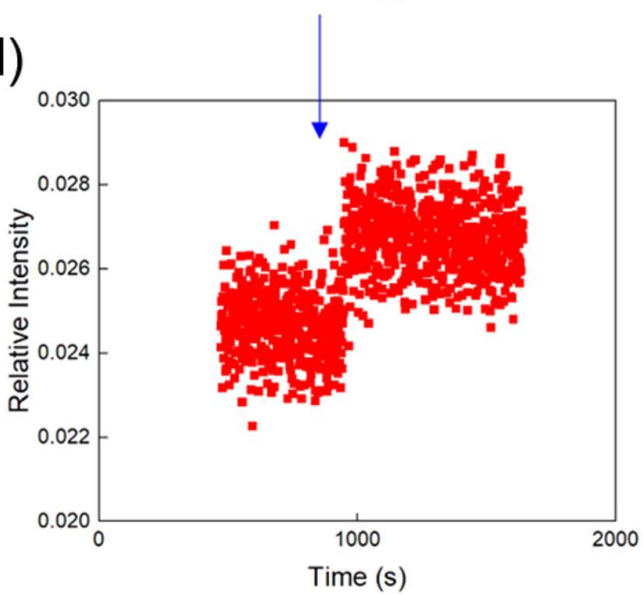

Figure S7. Exo III activity measured with a FAM-TAMRA pair in solution. (a) Fluorescence measurement with free Exo III enzymes. Initially, fluorescence is measured from $1 \mu M$ of FAM- 
DNA in solution. Upon addition of TAMRA-cDNA at $1 \mu \mathrm{M}$ (black arrow), the fluorescence intensity immediately drops. After a few minutes, we added $1 \mathrm{nM}$ Exo III enzymes into the solution (red arrow), which results in the increase of fluorescence intensity. (b) Fluorescence measurement in a similar experiment with particle-Exo III rather than free enzymes. The addition of polystyrene particle modified Exo III enzymes into the solution of FAM-DNA and TAMRA-cDNA ( $1 \mu \mathrm{M}$ each; green arrow) leads to the increase of fluorescence intensity. (c)(d) Fluorescence measurement with enzyme-particles encapsulated in giant vesicles with open origami pores. Black Arrow indicate the addition of TAMRA-cDNA into the solution of FAM-DNA ( $1 \mu \mathrm{M}$ each). Then, GUVs containing enzyme-particles are added to the solution (blue arrow). A distinct increase of the fluorescence intensity is observed. After a few minutes, particle-Exo III (without vesicles) are added directly to the solution (green arrow), which results in a further increase of the fluorescence intensity.

As Exo III can digest DNA in a duplex from the 3' end, it is important to measure how it will interact with origami channels. We incubated the particle-modified Exo III with DNA origami tiles for 15 mins, and then performed the AFM imaging. Note that 15-min incubation was used because it takes about 15 mins to encapsulate particle-Exo III in the giant vesicles with DNA origami channels. Figure S8 shows that most of the origami rectangles maintain their original shape. The results strongly suggest that the Exo III will not damage origami structures significantly under the experimental conditions.

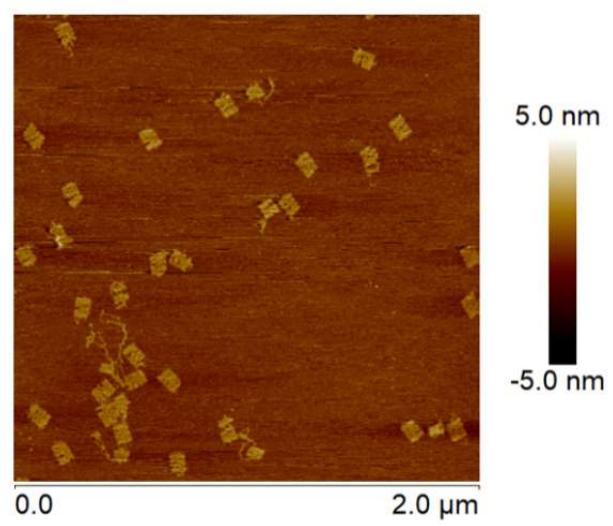

Figure S8. AFM image of DNA origami tiles after incubation with Exo III. Approximately $10 \mathrm{nM}$ DNA origami tiles were mixed with $0.1 \mathrm{nM}$ Exo III enzymes in $10 \mu \mathrm{L}$ TAEM buffer for 15 mins. The AFM image shows that most of the rectangular origami remains its original shape, indicating that this concentration of Exo III will do little damage to the origami.

\section{Immobilized giant vesicle shape change over time}

During the microscope observation, we find that several vesicles change shapes and sizes over time. Our observation suggests that the immobilized vesicles gradually flatten over time as additional binding occurs between the vesicles and the surface. Further, we also noted that the flow in the imaging chamber may cause some change in the vesicle shape as shown below in Figure S9. 

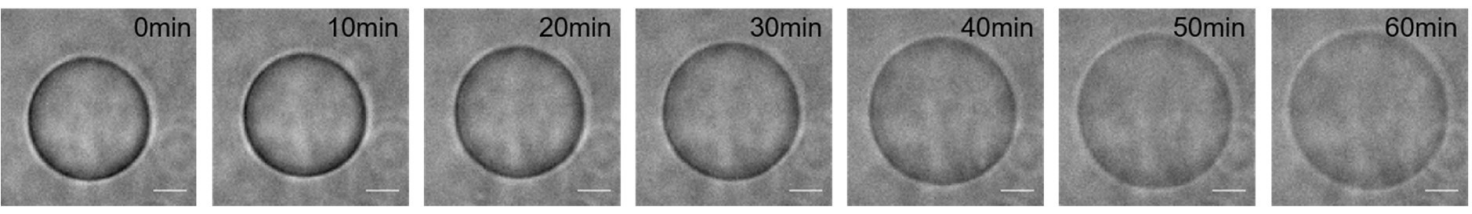

Figure S9. Shape change of a GUV during a one-hour period. Scale bar $=5 \mu \mathrm{m}$.

\section{Reversible aggregation behavior using DNA signals}

The reversible aggregation experiment using the SUV linkers was more efficient than using the hairpin strands, because it skips the process of (i) pore opening, (ii) hairpin penetration into the vesicle, (iii) cleavage by Exo III, and (iv) outflow of the transduced oligonucleotides (i.e., exposed SUV linkers) from the GUV. Therefore, we observed more drastic change in fluorescence intensity in a less amount of time. In the aggregation experiment using SUV linkers, approximately 30 mins were given for the SUVs to aggregate onto the giant vesicle, and about 20 mins for the small vesicles to dissociate from the GUV. With the hairpin strands, however, an hour was given for each step during the course of experiment. Figures S10 and S11 present additional reversible aggregation experiments (similar to Figure 5). To confirm that the hairpin strand itself cannot trigger the aggregation, we performed a control experiment that did not include the first step of adding cap releaser strands. Without cap releasers, the origami channels on the giant vesicles remain closed with the rectangular origami caps. The hairpin strands thus cannot enter the GUV to be converted into the SUV-linker strands. As a result, SUVs and hairpins cannot initiate the aggregation of small vesicles on the giant vesicle, as shown in Figure S12.

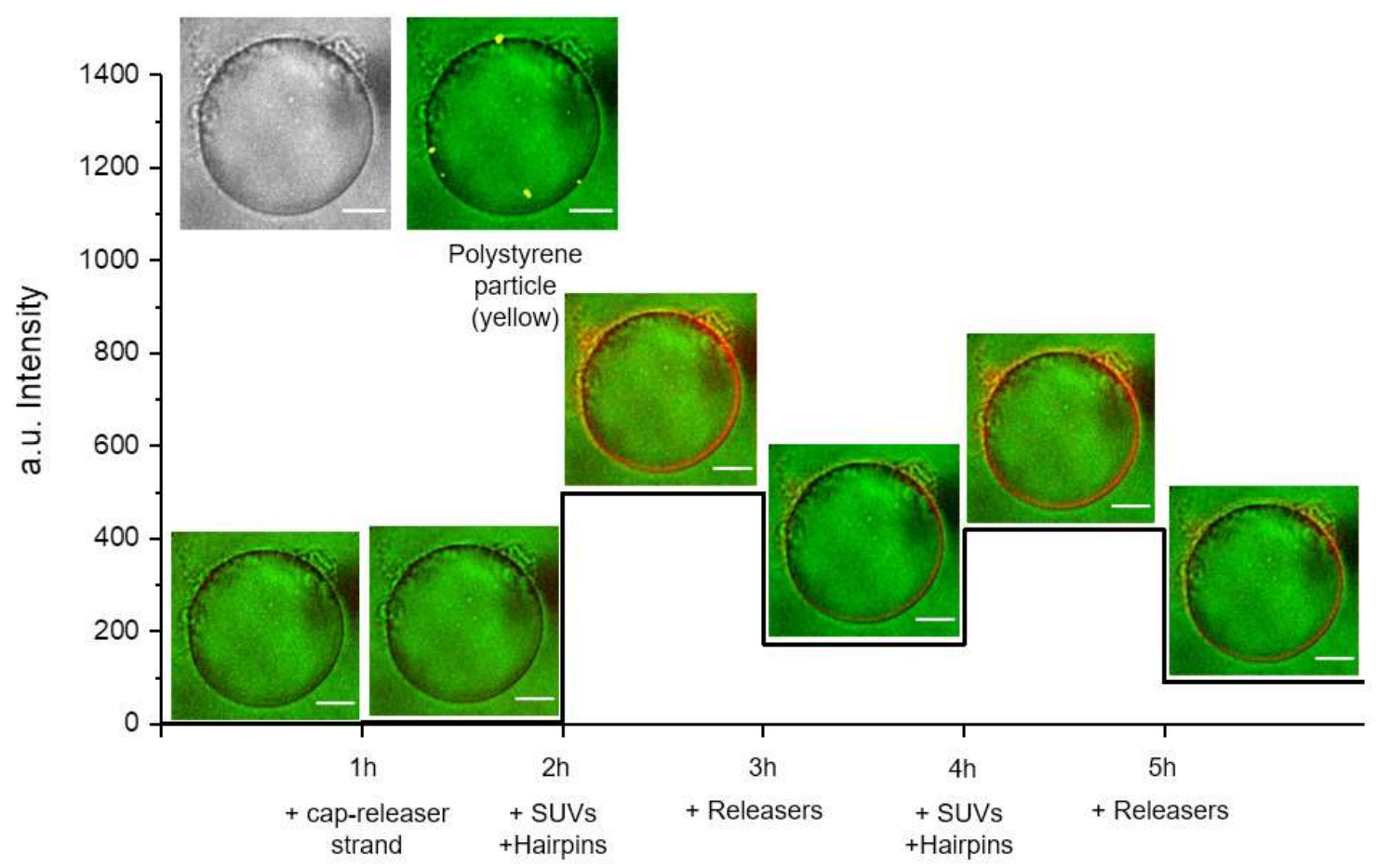

Figure S10. Additional reversible aggregation experiment with 2 cycles of adding SUVs, 
hairpin strands, and SUV releasers. For each step, we kept 1-hour incubation time, because the change of fluorescence intensity gradually stopped after 40 to 50 mins. Similar to Figure 5 , yellow dots in the top left image represents polystyrene particles functionalized with Exo III enzymes. Circular fluorescence ring indicates the aggregation of small vesicles on the surface of the giant vesicle.

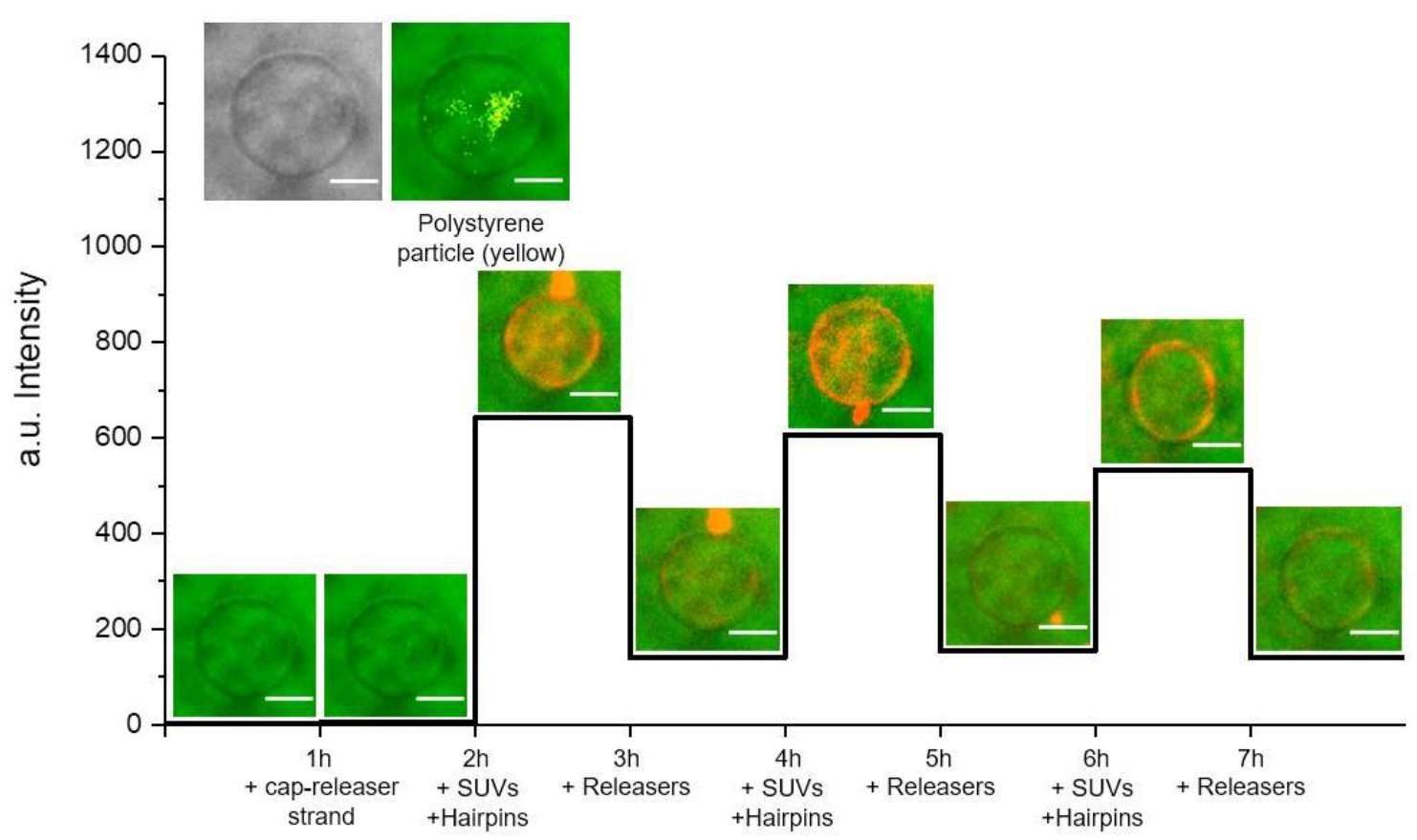

Figure S11. Additional aggregation experiment with hairpin signals. Fluorescence intensity increases were observed upon addition of SUVs and hairpin strands. Fluorescence drops were observed with presence of releasers. It is noticeable that the GUV immobilized on the glass surface gradually changed its shape from a sphere to ellipsoid. We suspect that this might be caused by the flow in the microfluidic channel. 


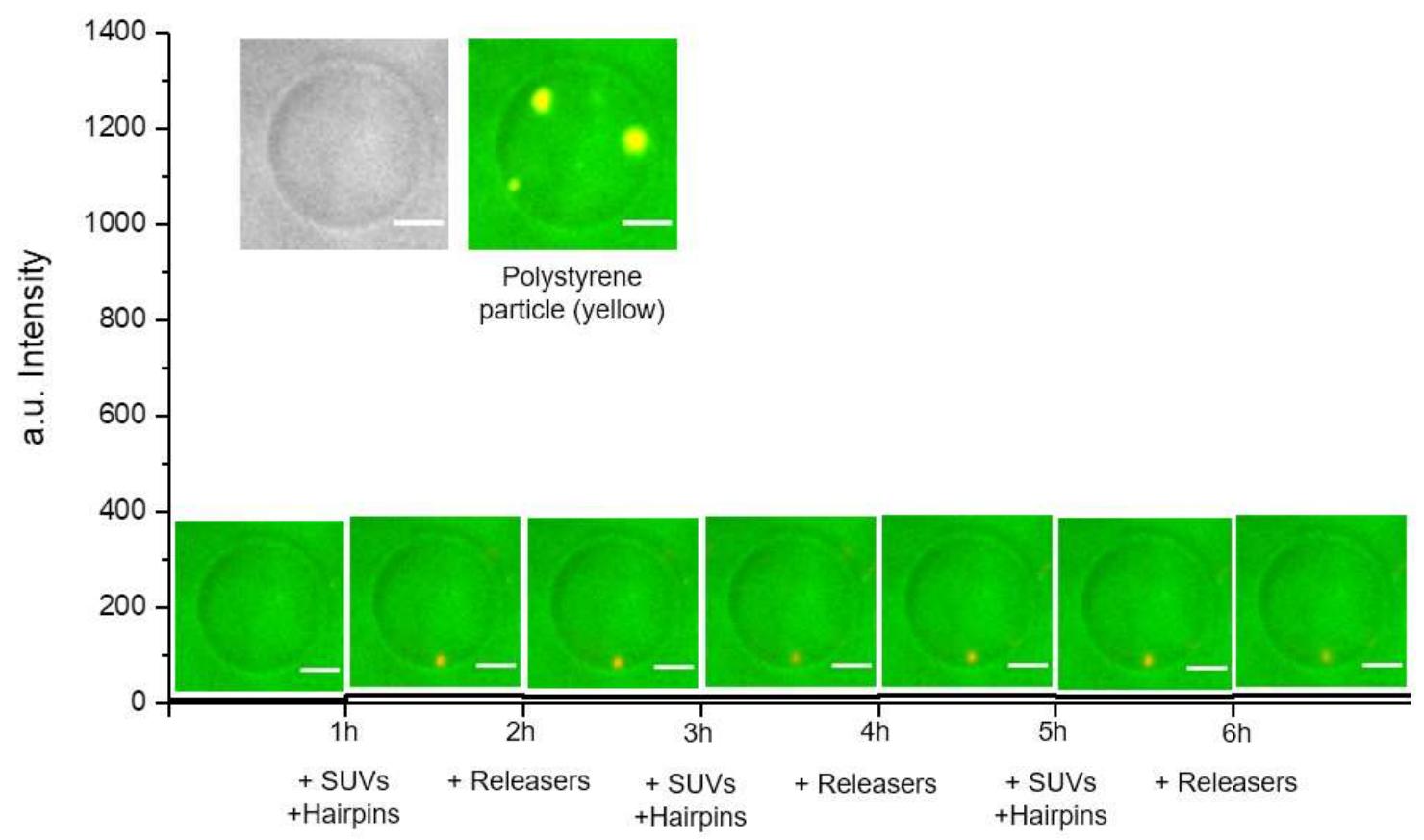

Figure S12. Control experiment without cap releaser strands. Small vesicles and hairpin strands were introduced into the microfluidic imaging chamber where a giant vesicle was immobilized with DNA pores closed with origami caps. Without adding cap releaser strands, the origami channels on the GUV remained closed; therefore, hairpin strands could not enter the GUV to become active SUV linker strand. As a result, we did not observe significant SUV binding on the GUV. There is only one spot that has nonspecifically bound SUV which was not removed by the SUV releaser strand.

\section{FRET measurement of vesicle aggregation}

Table S3. Sequences of DNA linker, releaser, and modified strands for FRET measurement of vesicle aggregation. SUV and GUV strands are modified with TAMRA and FAM, respectively. The TAMRA dye is attached on the 5' end of the SUV strand, while the FAM is attached on the 3' end of the GUV strand.

\begin{tabular}{|l|l|}
\hline Name & Sequence \\
\hline $\begin{array}{l}\text { TAMRA-SUV } \\
\text { strand }\end{array}$ & /56-TAMN/ TAA CAA CCA AAC CAT TTT T /3CholTEG/ \\
\hline $\begin{array}{l}\text { FAM-GUV } \\
\text { strand }\end{array}$ & /amine/GG ACA GAG TGA CAT C/36-FAM/ \\
\hline SUV linker & ATG GTT TGG TTG TTA GAT GTC ACT CTG TCC GAA TCA \\
\hline $\begin{array}{l}\text { Hairpin signal } \\
\text { containing } \\
\text { SUV linker }\end{array}$ & ATG GTT TGG TTG TTA GAT GTC ACT CTG TCC GAA TCA ACA TCT \\
\hline SUV releaser & TGA AAC CAA ACC AT \\
\hline
\end{tabular}

FRET experiments are designed to collect ensemble results of the vesicle aggregation. We 
attached the FAM fluorophore on the 3' end of the GUV strand and the TAMRA quencher on the 5' end of the SUV strand, as shown in Table S3. A schematic of FRET experiment is provided in Figure S13. Initially, the GUVs with FAM strands (GUV-strands with FAM moieties) and the SUVs with the TAMRA strands (SUV-strands with TAMRA groups) are mixed together at a concentration of $0.1 \mathrm{nM}$ in $150 \mu \mathrm{L}$ TAEM buffer. The SUVs and GUVs are not connected without linker strands (Figure S13a). Therefore, the fluorescence intensity of FAM from the GUVs remains high (Figure S14a) at $520 \mathrm{~nm}$ which is the emission peak of FAM fluorophore (also see Figure S4). This is because the FAM and TAMRA strands are not complementary, thus FAM and TAMRA are not in close proximity. Upon introduction of the $1 \mu \mathrm{L} 10 \mu \mathrm{M}$ linker strands, the SUVs aggregate onto the GUVs, resulting in a quenching of FAM fluorescence with TAMRA (Figure S13b). Thus, a significant fluorescence drop is observed as in Figure S14a. The addition of releaser strands will trigger release of the SUVs from the GUVs, causing the recovery of FAM fluorescence intensity (Figure S13c). Figure S14a shows the increase of FAM fluorescence upon addition of $1 \mu \mathrm{L} 1 \mathrm{mM}$ releaser strands, confirming the reversibility of aggregation process. Control experiments are shown in Figure 14b and 14c. In control experiments, no significant fluorescence changes are observed with the addition of buffer solution or random strands.
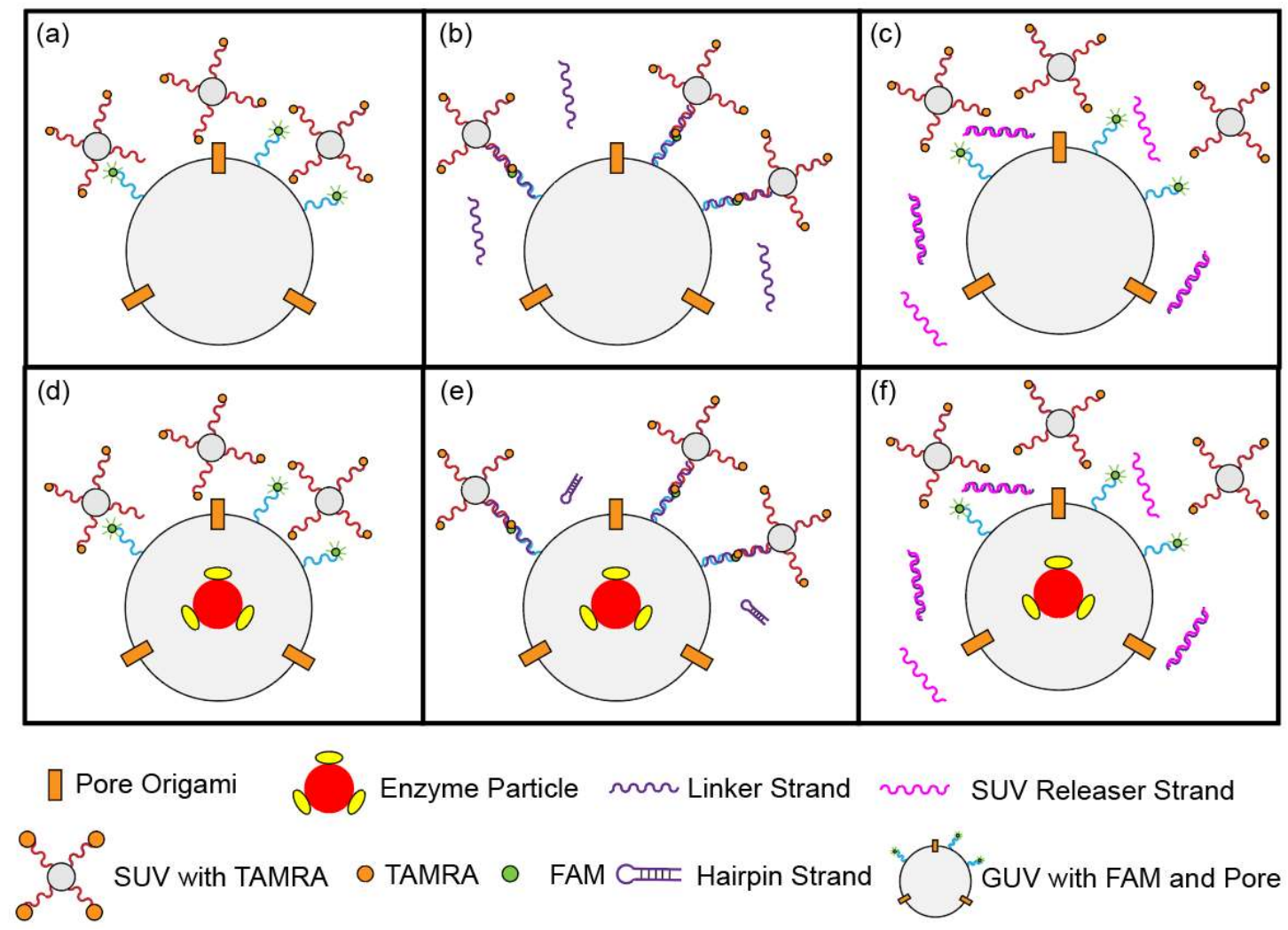

Figure S13. Schematic of FRET measurements for vesicle aggregation. Schematics in (a)-(c) demonstrate aggregation controlled by linker and releaser strands. (a) GUVs and SUVs are originally mixed. FAM fluorescence is observable as SUVs with TAMRA are not connected with GUVs. (b) Addition of linker strands induces the aggregation between SUVs and GUVs. The aggregation results in a quenching of FAM due to close contact with TAMRA. (c) 
Introduction of releaser strands results in a release of SUVs from GUVs, and thus, FAM fluorescence becomes prominent again. Schematics in (d)-(f) demonstrate aggregation triggered by hairpin strands. For simplicity, pores are used in the experiment without caps. (d) GUVs and SUVs are mixed together initially. However, they are not connected and strong FAM fluorescence is observed. (e) Hairpin strands are introduced and penetrate into the GUV, where they are cleaved by enzyme particles. The cleaved product is the same as the linker strand. The cleaved oligonucleotides move out of the GUV via origami pores and trigger SUV aggregation, resulting in a quenching of FAM fluorescence. (f) Addition of SUV-releaser strands causes the separation of SUVs and GUVs, which restores the FAM fluorescence.

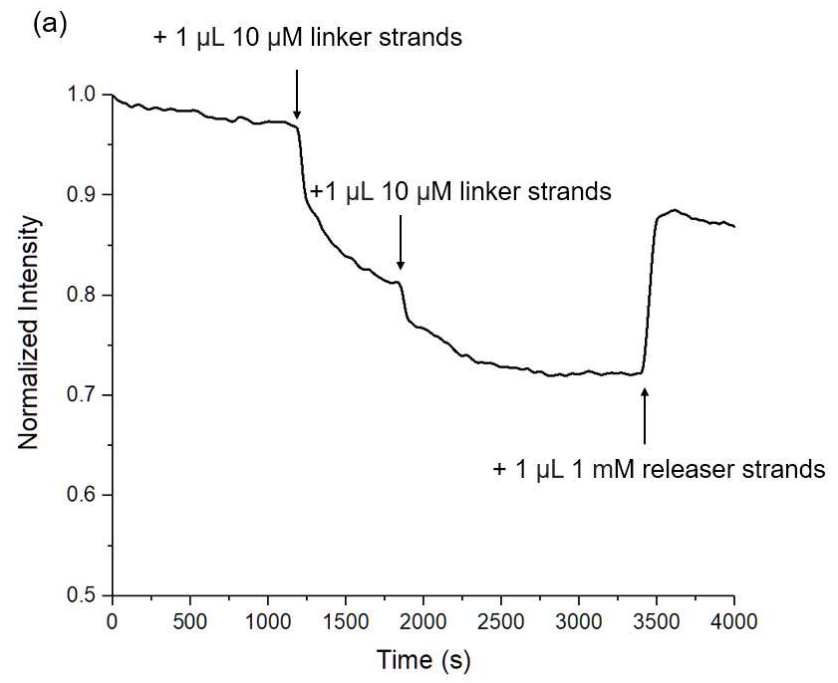

(b)

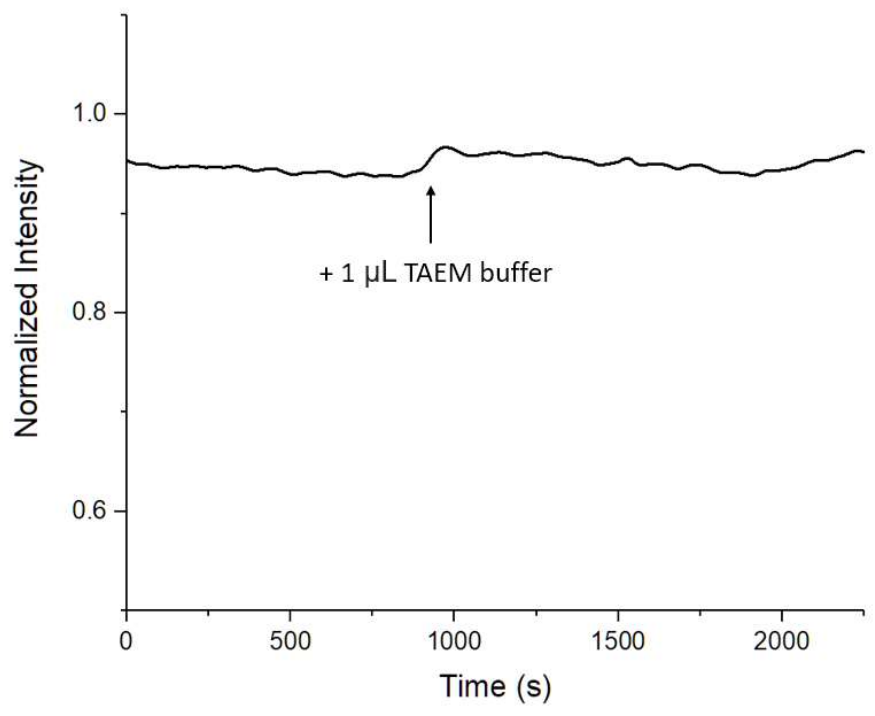




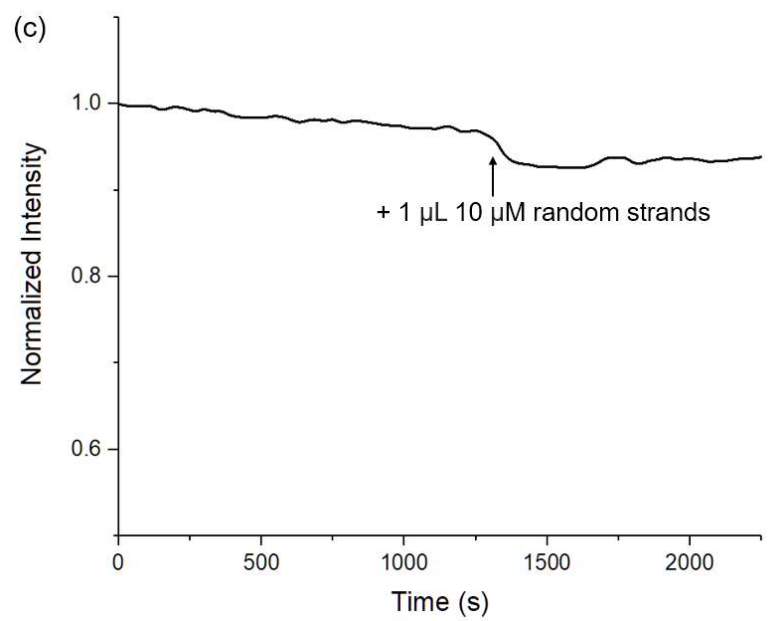

Figure S14. FRET measurement of vesicle aggregation programmed by linker and releaser strands, illustrated in Figure S13(a)-(c). Normalized fluorescence spectra of the FAM dye on the GUV-strands decorated on the GUV surface. (a) FAM-decorated GUVs and TAMRAfunctionalized SUVs are initially mixed and show strong fluorescence intensity. Addition of linker strands results in a significant fluorescence drop, confirming aggregations of the GUVs and SUVs. A fluorescence recovery is observed with the introduction of releaser strands, indicating the release of the SUVs from the GUVs. (b) Control experiment with buffer solution. Addition of buffer solution does not change fluorescence. (c) Control experiment with strands of random sequence, which does not alter the fluorescence intensity significantly.

We performed another set of FRET measurement to confirm the programmable vesicle aggregation with hairpin signals. Here, GUVs with the FAM strands contain EXO-III enzymemodified particles. For simplicity, the pores on the GUVs are not capped and allow hairpin strands to go through the pores. These GUVs are initially mixed with SUVs with the TAMRA strands, both at a concentration of $0.1 \mathrm{nM}$ in $150 \mu \mathrm{L}$ TAEM buffer. As illustrated in Figure S13d, the GUVs emit fluorescence from FAM as they are not connected with the SUVs decorated with TAMRA. Thus, a strong fluorescence signal is observed in Figure S15a. When hairpin strands are introduced, they will penetrate into the GUVs, become digested by the enzymes, translocate out of the GUVs via DNA pores, and then cause the SUV aggregation around the GUVs, ultimately quenching the FAM fluorescence (Figure S13e). As shown in Figure 15a, addition of $20 \mu \mathrm{L} 10 \mu \mathrm{M}$ hairpin strands result in the fluorescence drop. When $10 \mu \mathrm{L} 1 \mathrm{mM}$ releaser strands are added, they trigger disaggregation of the vesicles (Figure S13f), leading to a sudden increase of the FAM fluorescence (Figure S15a). In contrast, the control experiments with random strands or buffer do not induce any significant fluorescence changes as shown in Figure S15b and S15c. 

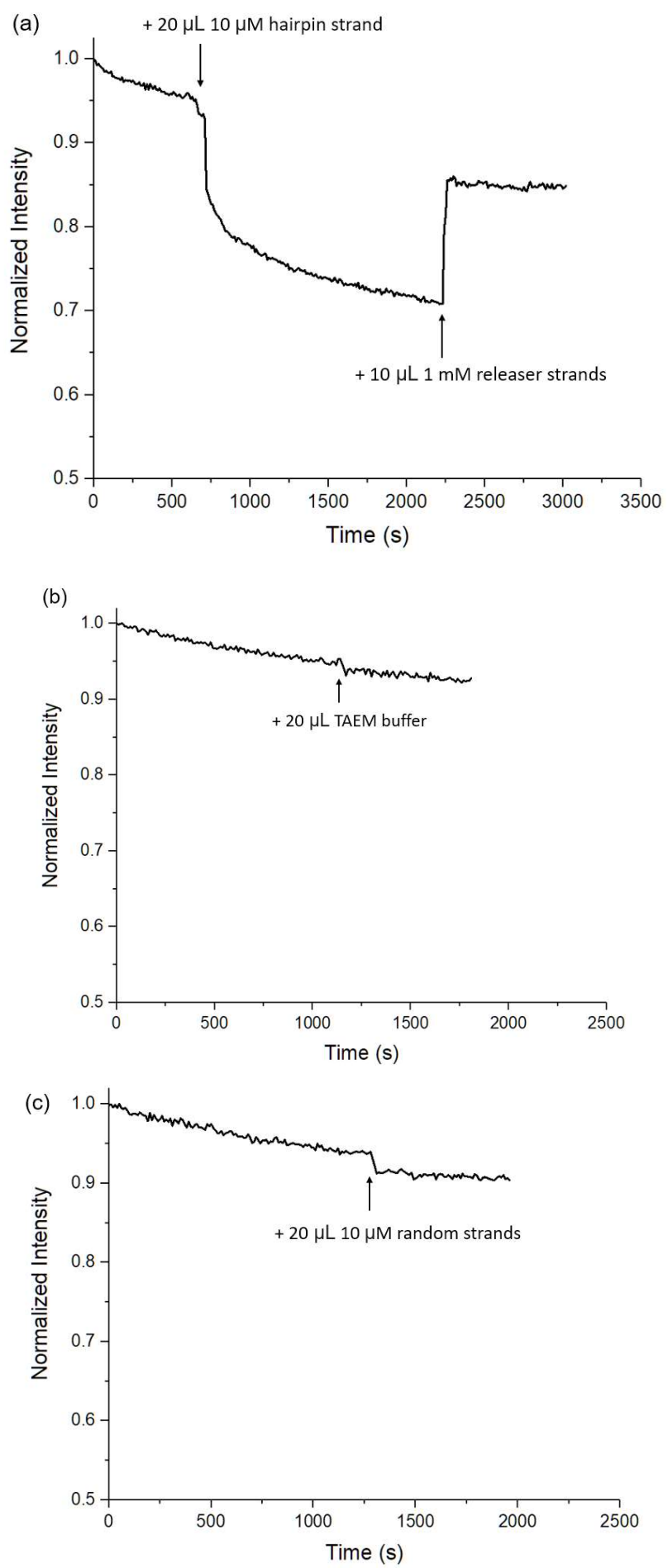

Figure S15. FRET measurement of vesicle aggregation with enzymes and hairpin strands. Normalized fluorescence spectra of the GUVs with the FAM strands, also containing EXO-IIImodified particles. (a) Initially, GUVs and SUVs are mixed and FAM fluorescence is observed due to no aggregation. The introduction of hairpin strands results in a significant fluorescence drop. The hairpin strands can be cleaved by the enzymes inside the GUVs and the cleaved 
product (which is the same as the linker strands in Figure 14) can trigger the aggregation of SUVs onto GUVs. After addition of releaser strands, a rapid recovery of fluorescence signal is observed, suggesting a disaggregation of vesicles. (b) Control experiment with buffer solution. Addition of buffer solution does not cause any significant fluorescence change. (c) Control experiment with random strands. No significant fluorescence intensity is observed. 


\section{References}

1. Chen, H.; Weng, T.-W.; Riccitelli, M. M.; Cui, Y.; Irudayaraj, J.; Choi, J. H., Understanding the mechanical properties of DNA origami tiles and controlling the kinetics of their folding and unfolding reconfiguration. Journal of the American Chemical Society 2014, 136, 6995-7005.

2. Li, R.; Chen, H.; Lee, H.; Choi, J. H., Elucidating the Mechanical Energy for Cyclization of a DNA Origami Tile. Applied Sciences 2021, 11, 2357.

3. Saccà, B.; Ishitsuka, Y.; Meyer, R.; Sprengel, A.; Schöneweiß, E. C.; Nienhaus, G. U.; Niemeyer, C. M., Reversible Reconfiguration of DNA Origami Nanochambers Monitored by Single-Molecule FRET. Angewandte Chemie International Edition 2015, 54, 3592-3597.

4. Müller, B. K.; Reuter, A.; Simmel, F. C.; Lamb, D. C., Single-pair FRET characterization of DNA tweezers. Nano Lett 2006, 6, 2814-20.

5. Thomsen, R. P.; Malle, M. G.; Okholm, A. H.; Krishnan, S.; Bohr, S. S.-R.; Sørensen, R. S.; Ries, O.; Vogel, S.; Simmel, F. C.; Hatzakis, N. S.; Kjems, J., A Large Size-selective DNA Nanopore with Sensing Applications. Nature Communications 2019, 10, 5655. 